\title{
Order/disorder phase transitions: the example of the Potts model
}

\author{
Hugo Duminil-Copin
}

\begin{abstract}
The critical behavior at an order/disorder phase transition has been a central object of interest in statistical physics. In the past century, techniques borrowed from many different fields of mathematics (Algebra, Combinatorics, Probability, Complex Analysis, Spectral Theory, etc) have contributed to a more and more elaborated description of the possible critical behaviors for a large variety of models (interacting particle systems, lattice spin models, spin glasses, percolation models). Through the classical examples of the Ising and Potts models, we survey a few recent advances regarding the rigorous understanding of such phase transitions for the specific case of lattice spin models. This review was written at the occasion of the Harvard/MIT conference Current Developments in Mathematics 2015.
\end{abstract}

\section{Contents}

1. Introduction 27

2. A warm-up: existence of an order/disorder phase transition 34

3. Critical behavior on $\mathbb{Z}^{2} \quad 42$

4. Critical behavior in dimension 3 and more $\quad 58$

References 68

\section{Introduction}

Lattice models have been introduced as discrete models for real life experiments and were later on found useful to model a large variety of phenomena and objects, ranging from ferroelectric materials to lattice gas. They also provide discretizations of Euclidean and Quantum Field Theories and are as such important from the point of view of theoretical physics. While the original motivation came from physics, they appeared as extremely complex and rich mathematical objects, whose study required the developments 
of important new tools that found applications in many other domains of mathematics.

The zoo of lattice models is very diverse: it includes models of spinglasses, quantum chains, random surfaces, spin systems, percolation models. Here, we focus on a smaller class of lattice models called spin systems. These systems are random collections of spin variables assigned to the vertices of a lattice. The archetypical example of such a model is provided by the Ising model, for which spins take value \pm 1 . This model was introduced by Lenz in 1920 to model the temperature, called Curie's temperature, above which a magnet looses its ferromagnetic properties.

This review describes a few aspects of the behavior of lattice spin models near their critical point. Emphasis is put on some probabilistic techniques developed to study these models.

1.1. Definition of the models. Let $G$ be a finite subgraph of the lattice $\mathbb{Z}^{d}$. The set of vertices and edges of $G$ are denoted respectively by $V(G)$ and $E(G)$. Let $\Sigma$ be a subset of $\mathbb{R}^{\nu}$, where $\nu \in \mathbb{N}^{*}$. A spin variable $\sigma_{x} \in \Sigma$ is attributed to each vertex $x \in V(G)$. A spin configuration $\sigma=$ $\left(\sigma_{x}: x \in V(G)\right) \in \Sigma^{V(G)}$ is given by the collection of all the spins.

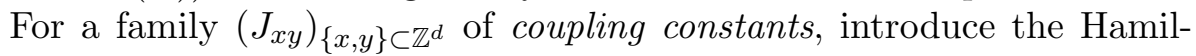
tonian of a spin configuration $\sigma$ defined by

$$
H_{G}^{\mathrm{free}}(\sigma):=-\sum_{\{x, y\} \subset V(G)} J_{x y}\left\langle\sigma_{x} \mid \sigma_{y}\right\rangle,
$$

where $\langle\cdot \mid \cdot\rangle$ denotes the standard scalar product on $\mathbb{R}^{\nu}$. The Gibbs measure on $G$ at inverse temperature $\beta$ with free boundary conditions is defined by the formula

$$
\mu_{G, \beta}^{\text {free }}[f]:=\frac{\int_{\Sigma^{V}(G)} f(\sigma) \exp \left[-\beta H_{G}^{\text {free }}(\sigma)\right] d \sigma}{\int_{\Sigma^{V}(G)} \exp \left[-\beta H_{G}^{\text {free }}(\sigma)\right] d \sigma}
$$

for every $f: \Sigma^{V(G)} \longrightarrow \mathbb{R}$, where

$$
d \sigma=\bigotimes_{x \in V(G)} d \sigma_{x}
$$

is a product measure whose marginals $d \sigma_{x}$ are identical copies of a reference finite measure $d \sigma_{0}$ on $\Sigma$. Note that if $\beta=0$, then spins are chosen independently according to the probability measure $d \sigma_{0} / \int_{\Sigma} d \sigma_{0}(\sigma)$.

A priori, $\Sigma$ and $d \sigma_{0}$ can be chosen to be arbitrary, thus leading to different examples of lattice spin models. The following (far from exhaustive list) of spin models already illustrates the vast variety of possibilities that such a formalism offers:

Ising model: $\Sigma=\{-1,1\}$ and $d \sigma_{0}$ is the counting measure on $\Sigma$. This model was introduced by Lenz in 1920 [Len20] to model 


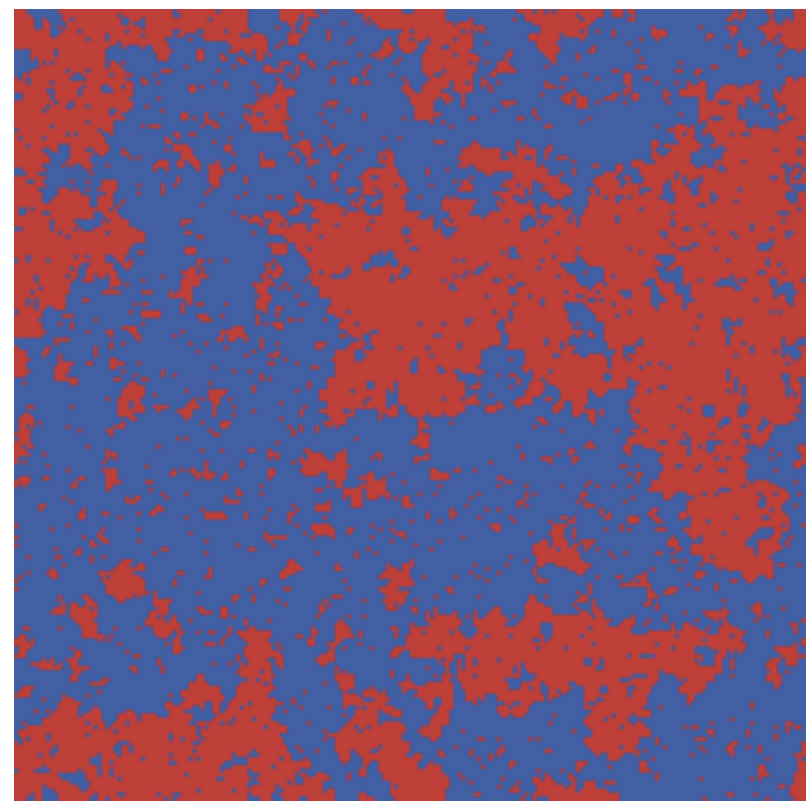

Figure 1. A simulation of the Ising model due to V. Beffara.
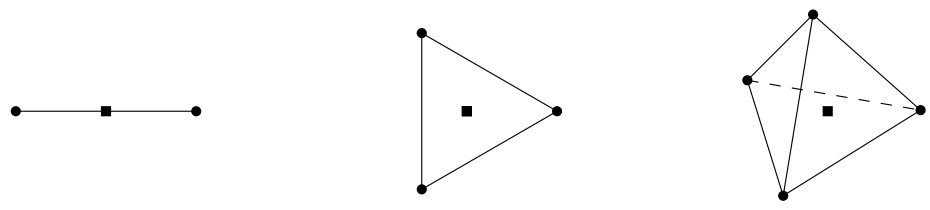

Figure 2. From left to right, $\mathbb{T}_{2}, \mathbb{T}_{3}$ and $\mathbb{T}_{4}$.

Curie's temperature for ferromagnets. It was studied in his $\mathrm{PhD}$ thesis by Ising [Isi25].

Potts model: $\Sigma=\mathbb{T}_{q}$ ( $q \geq 2$ is an integer $)$, where $\mathbb{T}_{q}$ is a simplex in $\mathbb{R}^{q-1}$ (see Fig. 2) containing $\overrightarrow{1}=(1,0, \ldots, 0)$ such that

$$
\left\langle\sigma_{x} \mid \sigma_{y}\right\rangle= \begin{cases}1 & \text { if } \sigma_{x}=\sigma_{y} \\ -\frac{1}{q-1} & \text { otherwise }\end{cases}
$$

and $d \sigma_{0}$ is the counting measure on $\Sigma$. This model was introduced by Potts [Pot52] following a suggestion of his adviser Domb. While the model received little attention early on, it became the object of great interest in the last forty years. Since then, mathematicians and physicists have been studying it intensively, and a lot is known on its rich behavior. It is now one of the foremost example of lattice models.

Spin $O(n)$ model: $\Sigma$ is the unit sphere in dimension $n$ and $d \sigma_{0}$ is the surface measure. This model was introduced by Stanley in [Sta68]. 


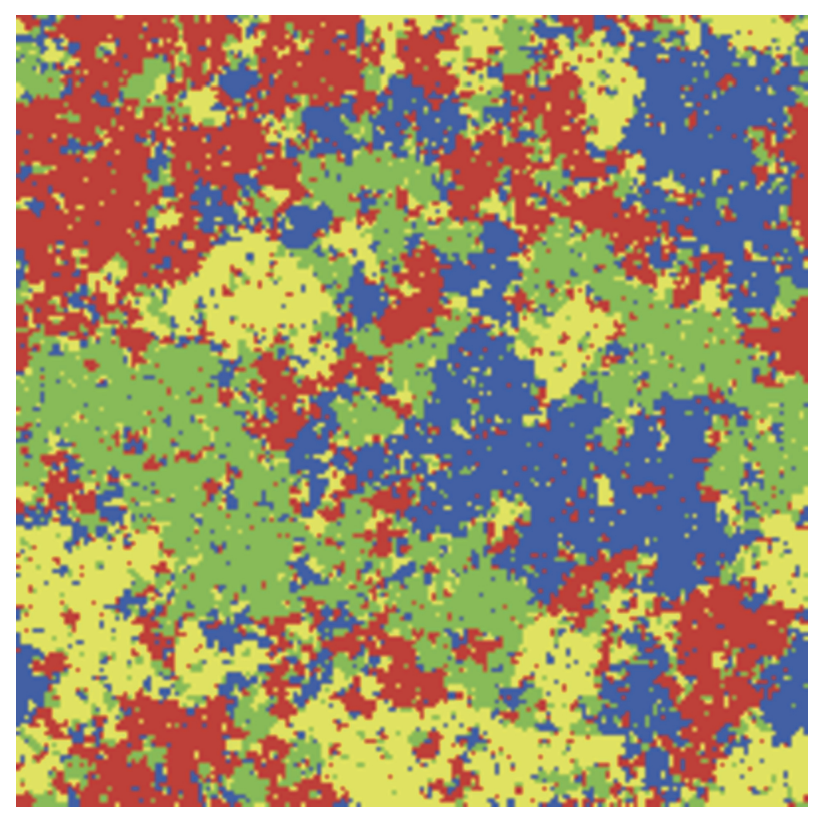

Figure 3. A simulation of the four-state Potts model due to V. Beffara.

This is yet another generalization of the Ising model (the case $n=1$ corresponds to the Ising model). The $n=2$ and $n=3$ models were introduced slightly before the general case and are called the $X Y$ and (classical) Heisenberg models respectively.

Discrete Gaussian Free Field (GFF): $\Sigma=\mathbb{R}$ and $d \sigma_{0}=$ $e^{-\sigma_{0}^{2} / 2} d \lambda\left(\sigma_{0}\right)$, where $d \lambda$ is the Lebesgue measure on $\mathbb{R}$. The discrete $\mathrm{GFF}$ is a lattice version of the continuum GFF, sometimes called Euclidean bosonic massless GFF, which is the starting point of many physical theories (for instance Liouville Quantum Gravity, to mention an example which is studied here in Cambridge).

The $\phi_{d}^{4}$ lattice model on $\mathbb{Z}^{d}: \Sigma=\mathbb{R}$ and $d \sigma_{0}=\exp \left(-a \sigma_{0}^{2}-\right.$ $\left.b \sigma_{0}^{4}\right) d \lambda\left(\sigma_{0}\right)$, where $a \in \mathbb{R}$ and $b \geq 0$. This model interpolates between the GFF corresponding to $a=1 / 2$ and $b=0$, and the Ising model corresponding to the limit as $b=-a / 2$ tends to $+\infty$.

We will assume that the model has nearest neighbor ferromagnetic coupling constants, meaning that $J_{x y}$ is zero except if $x$ and $y$ are neighbors, in which case $J_{x y}=J \geq 0$ (notice that we may assume without loss of generality that $J=1$, since multiplying $J$ by a constant corresponds to dividing $\beta$ by the same amount). The theories of non-ferromagnetic and non-nearest neighbors models (also called long-range models) are very interesting on their own right, nevertheless we prefer to reduce the scope of this lecture to the nearest neighbor ferromagnetic case to simplify the discussion. In the same spirit, and since the realm of continuous spin space is very different 
from the realm of discrete spin space, we choose to focus on the Potts model (which includes the Ising model as a special case).

An important notation. From now on, $\mu_{G, \beta, q}^{\text {free }}$ denotes the Gibbs measure for the nearest neighbor ferromagnetic $q$-state Potts model on $G$ with inverse temperature $\beta$ and free boundary conditions.

1.2. Continuous/discontinuous transition between an ordered and a disordered phase. Ferromagnetic models favor configurations in which spins point in the same direction. Furthermore, the parameter $\beta$ calibrates the strength of the interaction, or alternatively how much spins prefer to be aligned. For this reason, it does not come as a surprise that measures on very large graphs exhibit different behaviors at different inverse temperatures: for small $\beta$, the entropy should win on the energy, implying that spins should be roughly independent, while on the contrary for large values of $\beta$, the energy should be the most important factor, implying that spins should align in an ordered fashion.

Our goal is to study the appearance of this ordering, i.e. the appearance of a global alignment of the spins. In order to quantify this alignment, introduce the following random variable:

$$
\mathscr{M}(G):=\frac{1}{|V(G)|} \sum_{x \in V(G)} \sigma_{x} .
$$

In the case of the Potts model, the average of $\mathscr{M}(G)$ under the measure with free boundary conditions is always zero due to the symmetries of the spin space. In order to break the inherent symmetry, we introduce new boundary conditions as follows. Let

$$
H_{G}^{1}(\sigma):=H_{G}^{\mathrm{free}}(\sigma)-\sum_{x \in V(G), y \notin V(G)} J_{x y}\left\langle\sigma_{x} \mid \overrightarrow{1}\right\rangle
$$

and $\mu_{G, \beta, q}^{1}$ defined as $\mu_{G, \beta, q}^{\text {free }}$ with $H_{G}^{1}$ replacing $H_{G}^{\text {free }}$ (recall that $\overrightarrow{1}$ is the vector in $\Sigma \subset \mathbb{R}^{\nu}$ with first coordinate 1 and other coordinates 0$)$.

We may now speak of the average magnetization defined by

$$
m(G, \beta, q):=\mu_{G, \beta, q}^{1}[\mathscr{M}(G)]
$$

and of its limit

$$
m^{*}(\beta, q):=\lim _{n \rightarrow \infty} m\left(\Lambda_{n}, \beta, q\right),
$$

where $\Lambda_{n}:=\llbracket-n, n \rrbracket^{d}$. At this point, the quantity on the right-hand side of the previous equation is not a priori well defined since $m\left(\Lambda_{n}, \beta, q\right)$ could a priori fail to converge as $n$ tends to infinity. We will see in the next section that a cute coupling argument shows that the limit indeed exists and that the following occurs:

TheOREM 1.1. Let $d \geq 2$ and $q \geq 2$, there exists $\beta_{c}=\beta_{c}(d, q) \in(0, \infty)$ such that $m^{*}(\beta, q)=0$ if $\beta<\beta_{c}$ and $m^{*}(\beta, q) \neq 0$ if $\beta>\beta_{c}$. 

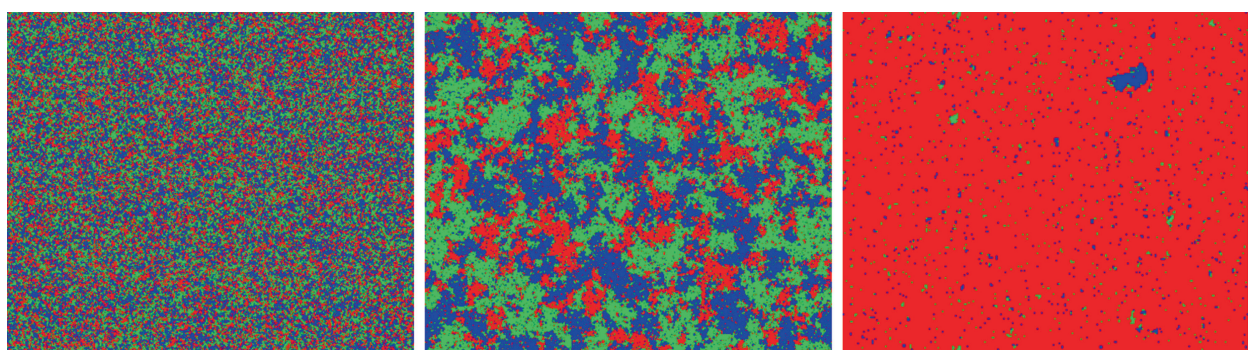

Figure 4. Simulations of three-state planar Potts model at subcritical, critical and supercritical temperatures.

The two ranges of $\beta$ for which $m^{*}(\beta, q)=0$ and $m^{*}(\beta, q) \neq 0$ are respectively called the disordered and ordered phases. The value $\beta_{c}$ is called the critical value, and the model is said to undergo an order/disorder phase transition.

Note that the previous theorem leaves the behavior at $\beta_{c}$ open: $m^{*}\left(\beta_{c}, q\right)$ may a priori be zero or non-zero. While this may appear like a detail, let us highlight that many aspects of the phase transition are governed by the behavior of the model at $\beta=\beta_{c}$, and that for this reason this is maybe the most interesting value of $\beta$ to study from the physics point of view. We will say that the phase transition is continuous if $m^{*}\left(\beta_{c}, q\right)=0$, and discontinuous otherwise.

Physicists have been interested in the behavior of the Potts model for a long time, and the following beautiful conjecture has emerged in the last fifty years.

CONJECTURE 1. Let $d \geq 2$ and $q \geq 2$. The phase transition of the nearest neighbor ferromagnetic q-state Potts model is continuous if $q \leq q_{c}(d)$ and discontinuous if $q>q_{c}(d)$, where

$$
q_{c}(d):= \begin{cases}4 & \text { if } d=2 \\ 2 & \text { if } d \geq 3\end{cases}
$$

For $d=2$, the conjecture is due to Baxter [Bax78]. For $d \geq 3$, the argument is based on considerations regarding the mean-field behavior of the model (see e.g. [BC03]). We postpone the discussion of known results to the beginning of each section (to maintain the suspense).

While the behavior of the model at the critical point of a discontinuous phase transition is fairly well understood (e.g. it features coexistence of different phases), the behavior at a continuous phase transition is much more mysterious and extraordinary rich. Indeed, continuous phase transitions are characterized by the absence of a correlation length. For this reason, the model at criticality may be studied via renormalization-type ideas and the large scale behavior of the model can be encoded via a limiting procedure (sometimes called taking the scaling limit) by Field Theories. The properties 

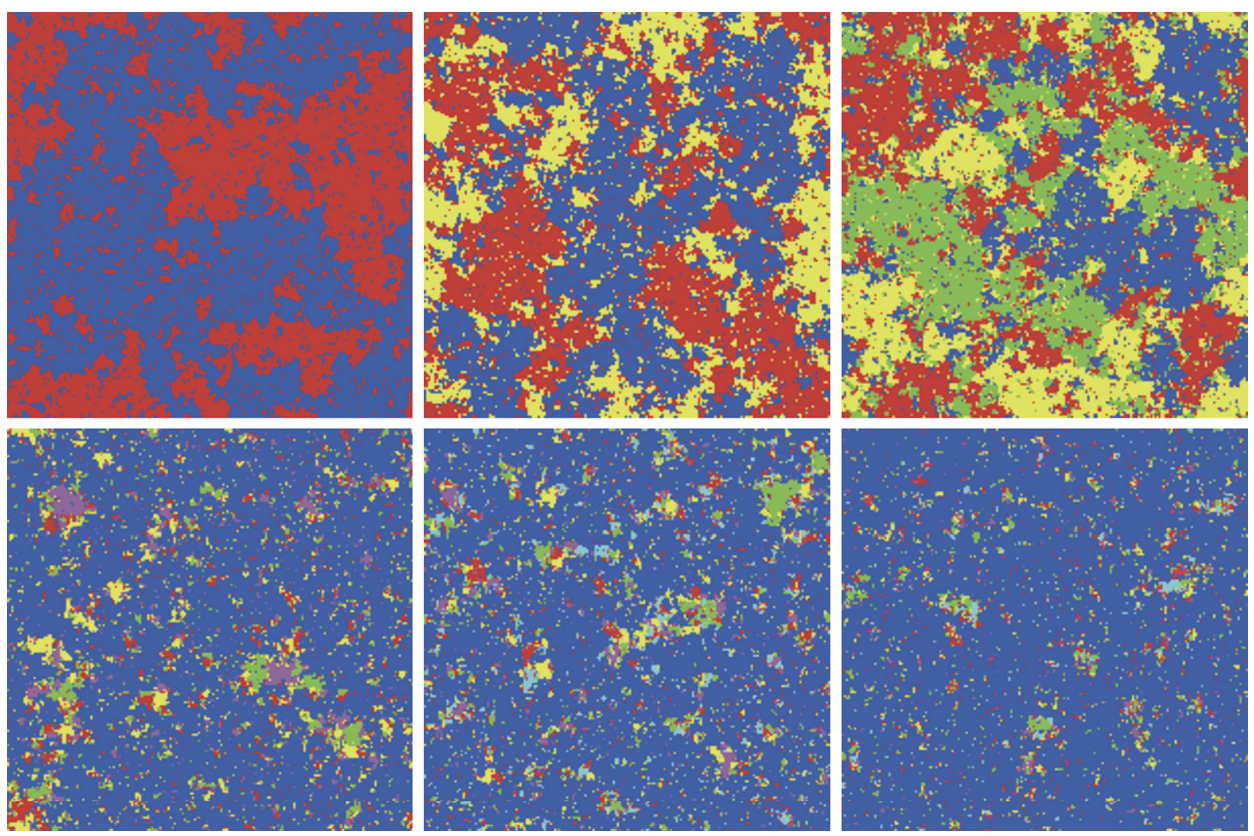

Figure 5. Simulations of the critical planar Potts model with $q$ equal to $2,3,4,5,6$ and 9 respectively. The behavior for $q \leq 4$ is clearly different from the behavior for $q>4$. In the first three pictures, each color (corresponding to each element of $\mathbb{T}_{q}$ ) seems to play the same role, while in the last three, one color wins over the other ones.

of these continuum versions of lattice models depend on the model and on the dimension.

If the model has a continuous phase transition in high enough dimension, then it exhibits its mean-field behavior, meaning that the critical exponents characterizing the phase transition are the same as those on the complete graph. For such dimensions, the Quantum Field Theory associated to the scaling limit of the critical models is trivial. For the Ising model, such a statement was proved by Aizenman and Fröhlich in dimension $d \geq 5[\mathbf{A i z 8 2}$, Frö82]. In dimension 4, critical exponents were proved to be equal to their mean-field value $[\mathbf{A F 8 8}$. The question of the triviality of the limiting Field Theory (i.e. the fact that the renormalized coupling constant vanishes) is still open.

On the contrary, the scaling limit of planar models at their critical point is not trivial (once again we restrict this discussion to continuous phase transitions). It is conjectured to be described by conformally invariant objects encoded by Conformal Field Theory [ISZ88]. Let us highlight the extraordinary scope of such a prediction: while the lattice version of the model is clearly invariant under the automorphisms of the lattice, its scaling limit is invariant under a much larger group of transformations, namely 
the conformal maps. This large group of symmetries enables physicists and mathematicians to derive very delicate properties of the model at criticality, and it is fair to say that the understanding of critical behavior in dimension two has greatly progressed in the last few decades thanks to Conformal Field Theory and its mathematical counterpart (for instance the theory of discrete holomorphic observables, the SLE machinery, random surfaces, etc).

The two previous paragraphs leave the case of dimension $d=3$, which is often the most interesting from the point of view of physics, open. There, new physical and mathematical ideas are required to construct the scaling limit of critical models, and this problem will probably occupy mathematicians and physicists for many years to come. Even at the physics level, the understanding is limited. Of course, conformal invariance may extend to arbitrary dimensions, yet the group of conformal transformations being reduced in dimension $d \geq 3$, the conclusions regarding the behavior of the scaling limit of the models are inevitably more limited (even though interesting developments using the so-called Conformal Bootstrap have shaken the physics community in the past few years, see e.g. $\left.\left[\mathbf{S F D}^{+} \mathbf{1 2}\right]\right)$.

\section{A warm-up: existence of an order/disorder phase transition}

As mentioned in the introduction, the existence of a phase transition itself is non-trivial. Indeed, several claims made in the previous section are not obvious:

- The quantity $m^{*}(\beta, q)$ is not clearly defined since the limit (as $n$ tends to infinity) of the quantity $m\left(\Lambda_{n}, \beta, q\right)$ may not exist.

- It is unclear whether $m^{*}(\beta, q)$ is equal to zero for small $\beta$, and is not equal to zero for large values of $\beta$. Interestingly, Ising wrongly predicted that the spontaneous magnetization would be equal to 0 for any values of $\beta$ independently of the dimension, based on the observation that it does so in dimension one (we will see later that he was completely wrong).

- While the intuition is convincing, it is a priori non trivial that there exists a critical value $\beta_{c}(q)$ separating an ordered from a disordered phase. It may very well be that the model alternates between ordered and disordered phases when $\beta$ increases. Let us illustrate the difficulty through the example of the Ising model. In order to rule out the possibility of alternating phases, we could prove that $\frac{d}{d \beta} m(G, \beta, 2)$ which is equal to

$$
\frac{1}{|V(G)|} \sum_{x \in V(G)} \sum_{\{a, b\} \in E(G)} \mu_{G, \beta, 2}^{1}\left[\sigma_{x} \sigma_{a} \sigma_{b}\right]-\mu_{G, \beta, 2}^{1}\left[\sigma_{x}\right] \mu_{G, \beta, 2}^{1}\left[\sigma_{a} \sigma_{b}\right],
$$

must necessarily be positive. This would readily imply that the spontaneous magnetization would also be. The positivity would follow from

$$
\mu_{G, \beta, 2}^{1}\left[\sigma_{x} \sigma_{a} \sigma_{b}\right]-\mu_{G, \beta, 2}^{1}\left[\sigma_{x}\right] \mu_{G, \beta, 2}^{1}\left[\sigma_{a} \sigma_{b}\right] \geq 0
$$



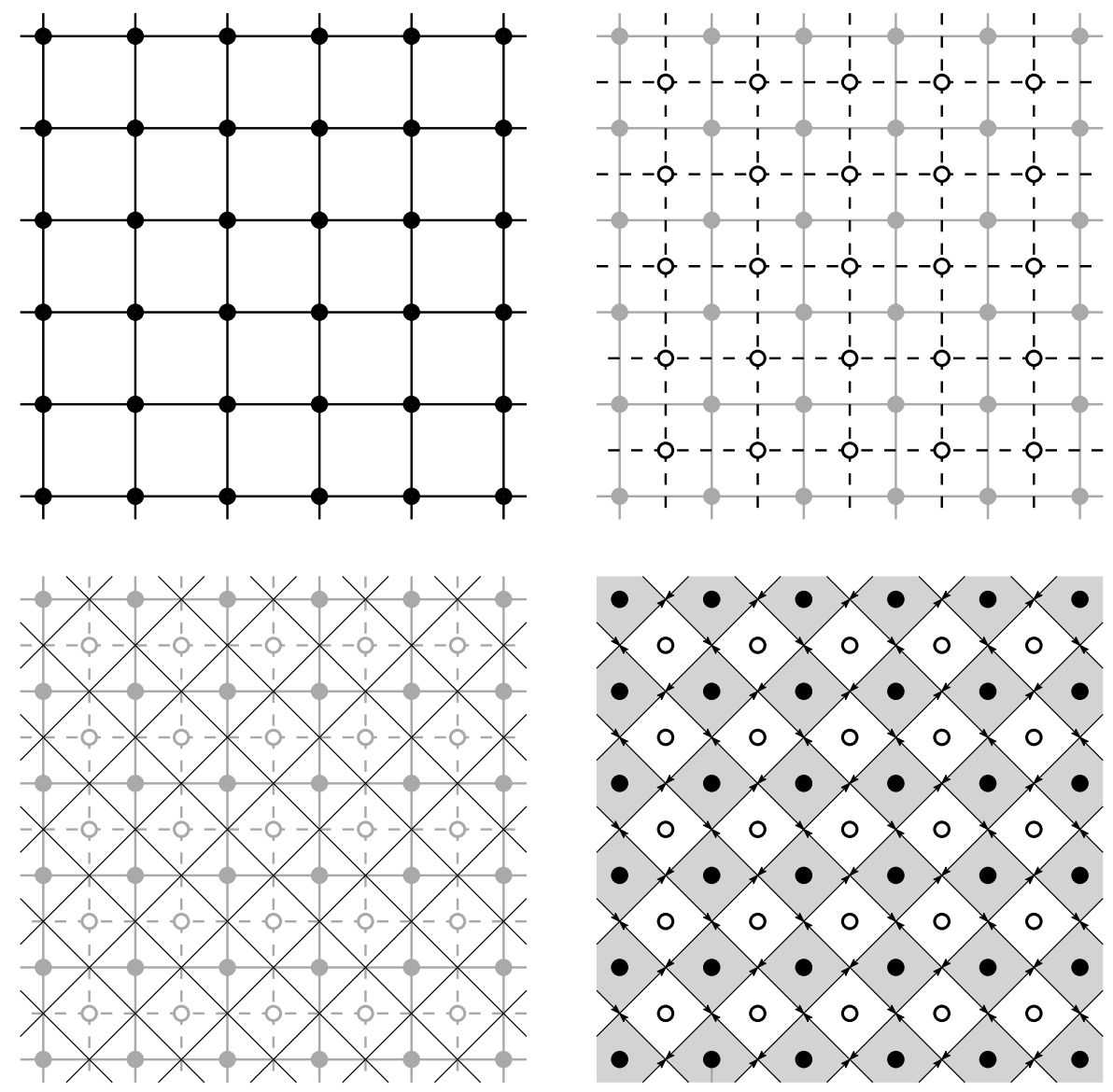

Figure 6 . The square lattice (top left), its dual lattice (top right), its medial lattice (bottom left) and a natural orientation on the medial lattice (bottom right).

for any fixed $a$ and $b$, an inequality which is far from obvious (it is called the second Griffiths' inequality).

Theorem 2.1 (Folklore). Let $d \geq 2$ and $q \geq 2$. For any $\beta>0$, there exists a probability measure $\mu_{\beta, q}^{1}$ on spin configurations on $\mathbb{Z}^{d}$ such that

$$
\lim _{G \nearrow \mathbb{Z}^{d}} \mu_{G, \beta, q}^{1}=\mu_{\beta, q}^{1}
$$

where the convergence is the weak convergence for measures.

Furthermore,

$$
\lim _{n \rightarrow \infty} m\left(\Lambda_{n}, \beta, q\right)=\lim _{n \rightarrow \infty} \mu_{\Lambda_{n}, \beta, q}^{1}\left(\sigma_{0}\right)=\mu_{\beta, q}^{1}\left(\sigma_{0}\right)
$$

and

$$
\beta_{c}(q)=\inf \left\{\beta>0: \mu_{\beta, q}^{1}\left(\sigma_{0}\right) \neq 0\right\}=\sup \left\{\beta>0: \mu_{\beta, q}^{1}\left(\sigma_{0}\right)=0\right\}
$$

belongs to $(0, \infty)$. 
With the notation of the previous theorem, we see that $m^{*}(\beta, q)$ can be interpreted as the average spin at 0 for the infinite-volume measure $\mu_{\beta, q}^{1}$.

Proving all these properties using only the formalism of lattice spin models introduced in the previous section is not straightforward. It was done using correlation inequalities in the case of the Ising model, but remained open for the Potts model until the discovery by Fortuin and Kasteleyn [FK72] of a deep link between spin models and percolation-type models. The following sections are devoted to the description of this connection.

\subsection{A coupling between the Potts model and a percolation model.}

2.1.1. The Fortuin-Kasteleyn percolation. A percolation configuration $\omega=\left(\omega_{e}: e \in E(G)\right)$ is an element of $\{0,1\}^{E(G)}$. If $\omega_{e}=1$, the edge $e$ is said to be open, otherwise $e$ is said to be closed. A configuration $\omega$ can be seen as a subgraph of $G$ with $V(\omega)=V(G)$ and $E(\omega)=\left\{e \in E(G): \omega_{e}=1\right\}$. A percolation model is given by a distribution on percolation configurations on $G$.

In order to study the connectivity properties of the (random) graph $\omega$, we introduce some notation. A cluster is a maximal connected component of the graph $\omega$ (it may be an isolated vertex). Two vertices $x$ and $y$ are connected in $\omega$ if they are in the same cluster. We denote this event by $x \longleftrightarrow y$. For $A, B \subset \mathbb{Z}^{d}$, set $A \longleftrightarrow B$ if there exists a vertex of $A$ connected to a vertex of $B$. We also allow ourselves to consider $B=\infty$, in which case we mean that a vertex in $A$ is in an infinite cluster.

The simplest example of percolation model is provided by Bernoulli percolation: each edge is open with probability $p$, and closed with probability $1-p$, independently of the state of other edges. This model was introduced by Broadbent and Hammersley in [BH57] and has been one of the most studied probabilistic model. We refer to [Gri99] for a book on the subject.

Here, we will be interested in a slightly more complicated percolation model, named the Fortuin-Kasteleyn percolation (also called FK percolation or random-cluster model), which is a percolation model in which the states open/closed of edges depend on each others.

Let $G$ be a finite subgraph of $\mathbb{Z}^{d}$. Let $o(\omega)$ and $c(\omega)$ denote the number of open and closed edges of $\omega$. The boundary conditions $\xi$ are given by a partition $P_{1} \sqcup \cdots \sqcup P_{k}$ of

$$
\partial G:=\left\{x \in V(G): \exists y \notin V(G),\{x, y\} \in E\left(\mathbb{Z}^{d}\right)\right\}
$$

and $k_{\xi}(\omega)$ denotes the number of clusters in the graph $\omega$ where clusters intersecting each $P_{i}$ are counted as one.

Definition 2.2. The probability measure $\phi_{G, p, q}^{\xi}$ of the FK percolation on $G$ with edge-weight $p \in[0,1]$, cluster-weight $q>0$ and boundary conditions 
$\xi$ is defined by

$$
\phi_{G, p, q}^{\xi}[\{\omega\}]:=\frac{p^{o(\omega)}(1-p)^{c(\omega)} q^{k_{\xi}(\omega)}}{Z_{G, p, q}^{\xi}}
$$

for every configuration $\omega \in\{0,1\}^{E(G)}$. The constant $Z_{G, p, q}^{\xi}$ is a normalizing constant, referred to as the partition function, defined in such a way that the sum over all configurations equals 1.

For $q=1$, FK percolation corresponds to Bernoulli percolation.

Let us provide two examples of boundary conditions. The free boundary conditions correspond to the partition composed of singletons only. Then $k_{\text {free }}(\omega)$ simply denotes the number of clusters in $\omega$. The wired boundary conditions correspond to the partition $\{\partial G\}$ and $k_{\text {wired }}(\omega)$ is the number of clusters obtained if all clusters touching the boundary are counted as 1 .

2.1.2. The coupling. Consider an integer $q \geq 2$ and let $G$ be a finite graph. Assume that a configuration $\omega \in\{0,1\}^{E(G)}$ is given. One can deduce a spin configuration $\sigma \in \mathbb{T}_{q}^{V(G)}$ by assigning uniformly and independently to each cluster $\mathscr{C}$ of $\omega$ a spin $\sigma_{\mathscr{C}} \in \mathbb{T}_{q}$, except for the clusters intersecting the boundary $\partial G$ which are automatically associated to the spin $\overrightarrow{1}$. We then define $\sigma_{x}$ to be equal to $\sigma_{\mathscr{C}}$ for every $x \in \mathscr{C}$. Note that all the vertices in the same cluster automatically receive the same spin.

Proposition 2.3. Fix an integer $q \geq 2$. Let $p \in(0,1)$ and $G \subset \mathbb{Z}^{d} a$ finite graph. If the configuration $\omega$ is distributed according to $\phi_{G, p, q}^{\text {wired }}$, then the spin configuration $\sigma$ is distributed according to the q-state Potts measure $\mu_{G, \beta, q}^{1}$, where

$$
\beta=\beta(p, q)=-\frac{q-1}{q} \ln (1-p) .
$$

Since the proof is elementary, we include it here.

Proof. Let $\Omega$ be the space of pairs $(\omega, \sigma)$ with $\omega \in\{0,1\}^{E(G)}$ and $\sigma \in \mathbb{T}_{q}^{V(G)}$, with the property that for any edge $e=\{x, y\}$,

$$
\omega_{e}=1 \quad \Rightarrow \quad \sigma_{x}=\sigma_{y}
$$

Consider a measure $\mathbf{P}$ on $\Omega$, where $\omega$ is a percolation configuration with wired boundary conditions and $\sigma$ is the corresponding spin configuration constructed as explained above. Then, for $(\omega, \sigma)$, we have:

$$
\begin{aligned}
\mathbf{P}[(\omega, \sigma)] & =\frac{1}{Z_{G, p, q}^{\text {wired }}} p^{o(\omega)}(1-p)^{c(\omega)} q^{k_{\text {wired }}(\omega)} \cdot q^{-k_{\text {wired }}(\omega)+1} \\
& =\frac{q}{Z_{G, p, q}^{\text {wired }}} p^{o(\omega)}(1-p)^{c(\omega)} .
\end{aligned}
$$

(The additive constant 1 is due to the fact that the spin of the clusters of $\omega$ touching the boundary is necessarily $\overrightarrow{1}$.) 
Now, we construct another measure $\widetilde{\mathbf{P}}$ on $\Omega$ as follows. Let $\tilde{\sigma}$ be a spinconfiguration distributed according to $\mu_{G, \beta, q}^{1}$, where $\exp \left[-\frac{q}{q-1} \beta\right]=1-p$. We deduce $\tilde{\omega}$ from $\tilde{\sigma}$ by independently opening edges between neighboring vertices having the same spins with probability $p$. By definition, edges between vertices having different spins remain automatically closed. Then, for any $(\tilde{\omega}, \tilde{\sigma})$,

$$
\widetilde{\mathbf{P}}[(\tilde{\omega}, \tilde{\sigma})]=\frac{\exp \left[-\frac{q}{q-1} \beta r(\tilde{\sigma})\right] p^{o(\tilde{\omega})}(1-p)^{c(\tilde{\omega})-r(\tilde{\sigma})}}{Z}=\frac{p^{o(\tilde{\omega})}(1-p)^{c(\tilde{\omega})}}{Z},
$$

where $r(\tilde{\sigma})$ is the number of edges between vertices with different spins, and $Z$ is a normalizing constant.

In conclusion, $\mathbf{P}$ and $\widetilde{\mathbf{P}}$ are two probability measures on $\Omega$ assigning the same weights to configurations, they are therefore equal. Since the first marginal of $\mathbf{P}(=\widetilde{\mathbf{P}})$ is $\phi_{G, p, q}^{\text {wired }}$ and the second of $\widetilde{\mathbf{P}}(=\mathbf{P})$ is $\mu_{G, \beta, q}^{1}$, we obtain the result.

The coupling immediately extends to the FK percolation and the Potts model with free boundary conditions (we leave the question of what must be done with clusters touching the boundary as an exercise).

This coupling provides us with a dictionary between the properties of the FK percolation and the Potts model. In order to illustrate this fact, let us mention one consequence.

Corollary 2.4. Let $d, q \geq 2$. Let $G$ be a finite subgraph of $\mathbb{Z}^{d}$. Let $\beta>0$ and $p \in[0,1]$ be connected by (2.2). We have that for any $x \in V(G)$,

$$
\left.\mu_{G, \beta, q}^{1}\left[\sigma_{x}\right]=\mathbf{E}\left[\sigma_{x} \mathbf{1}_{\{x \stackrel{\omega}{\longleftrightarrow} \partial G\}}\right]+\mathbf{E}\left[\sigma_{x} \mathbf{1}_{\{x \longleftrightarrow} \stackrel{\omega}{\longleftrightarrow} \partial G\right\}\right]=\phi_{G, p, q}^{\text {wired }}[x \longleftrightarrow \partial G] \cdot \overrightarrow{1} .
$$

Proof. Simply use that $\sigma_{x}$ must be equal to $\overrightarrow{1}$ if $x$ is connected to $\partial G$, and $\sigma_{x}$ is uniformly chosen among spins in $\mathbb{T}_{q}$ otherwise.

This result will be used in the next sections to relate the phase transitions of the Potts model and of FK percolation. As a side remark, note that we just proved that $\left\langle\mu_{G, \beta, q}^{1}\left[\sigma_{x}\right] \mid \overrightarrow{1}\right\rangle$ is non-negative.

2.2. Phase transition and critical point. We now wish to prove that the Potts model undergoes a phase transition in dimension $d \geq 2$, meaning that $\beta_{c}(q, d)$ exists and belongs to $(0, \infty)$.

2.2.1. Ordering in the FK percolation. One of the advantages of percolation configurations compared to spin configurations is that $\{0,1\}^{E(G)}$ is naturally ordered (simply say that $\omega \leq \omega^{\prime}$ if any edge $e \in E(G)$ satisfies $\left.\omega_{e} \leq \omega_{e}^{\prime}\right)$. An event $A$ is increasing if any $\omega^{\prime} \geq \omega$ with $\omega \in A$ satisfies that $\omega^{\prime} \in A$. Let us mention briefly a few properties of FK percolation concerning increasing events. We refer to [Gri06, Dum13] for proofs.

Fix $q \geq 1$ and $G$ a finite graph. Let $p \leq p^{\prime}$ and $\xi \leq \psi$ (meaning that any element of the partition $\xi$ is included in an element of the partition $\psi$ ). Also 
consider two increasing events $A$ and $B$, then

$$
\begin{array}{lr}
\phi_{G, p, q}^{\xi}[A \cap B] \geq \phi_{G, p, q}^{\xi}[A] \phi_{G, p, q}^{\xi}[B] & \text { (FKG inequality) } \\
\phi_{G, p^{\prime}, q}^{\xi}[A] \geq \phi_{G, p, q}^{\xi}[A] & \text { (monotonicity in } p \text { ) } \\
\phi_{G, p, q}^{\psi}[A] \geq \phi_{G, p, q}^{\xi}[A] & \text { (comparison of boundary conditions). }
\end{array}
$$

One should really emphasize the fact that the cluster weight $q$ must be larger than or equal to 1 . These properties all fail for $q<1$. The model is actually expected to be negatively correlated in this context, see [Gri06, Section 3.9] for a discussion.

2.2.2. Phase transition in the FK percolation model. Phase transitions are properties of infinite-volume systems. For FK percolation, the definition of a measure on $\mathbb{Z}^{d}$ is not direct since one cannot count the number of open or closed edges on $\mathbb{Z}^{d}$ (they could be and would be infinite). We thus define infinite-volume measures by taking a sequence of measures on larger and larger boxes $\Lambda_{n}$, where $n \geq 1$. The ordering between boundary conditions can be used to show that the sequences of measures $\left(\phi_{p, q, \Lambda_{n}}^{\text {wired }}\right)_{n \geq 0}$ and $\left(\phi_{p, q, \Lambda_{n}}^{\text {free }}\right)_{n \geq 0}$ converge weakly to measures $\phi_{p, q}^{\text {wired }}$ and $\phi_{p, q}^{\text {free }}$ on $\{0,1\}^{E\left(\mathbb{Z}^{d}\right)}$, called the infinite-volume measures with wired and free boundary conditions respectively.

One warning: while boundary conditions cannot be defined as a partition of the boundary in infinite volume, one still needs to keep track of the dependency on boundary conditions for finite-volume measures when constructing

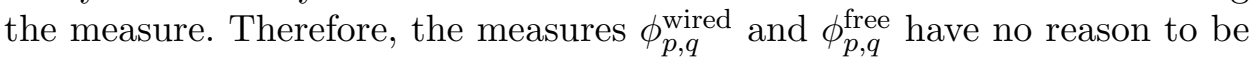
the same (we will see examples of values of $p$ and $q$ for which they are in fact different). In addition to this, one may imagine other infinite-volume measures obtained via limits of measures on finite graphs with arbitrary (and possibly random) boundary conditions. Nonetheless, the measures $\phi_{p, q}^{\text {wired }}$ and $\phi_{p, q}^{\text {free }}$ play very specific roles in the theory. First, they are invariant under translations and ergodic. Second, they are extremal infinite-volume measures, in the sense that any infinite-volume measure $\phi$ (see e.g. [Dum13, Definition 4.24] and references therein for a formal definition) with parameters $p$ and $q \geq 1$ satisfies

$$
\phi_{p, q}^{\text {free }}[A] \leq \phi[A] \leq \phi_{p, q}^{\text {wired }}[A]
$$

for any increasing events $A$. Third, an abstract theorem based on the convexity of the free energy (see [Dum13, Theorem 4.30]) shows that for a fixed $q \geq 1, \phi_{p, q}^{\text {free }}=\phi_{p, q}^{\text {wired }}$ (and therefore the infinite-volume measure is unique at this value of $p$ ) for all but possibly countably many values of $p$.

Let us make an additional remark. The properties of finite-volume measures (FKG inequality, monotonicity, ordering between boundary conditions) extend to infinite volume in a straightforward fashion.

We are now in a position to discuss the phase transition for the FK percolation. 
Theorem 2.5. For $q, d \geq 1$, there exists a critical point $p_{c}=p_{c}(q, d) \in$ $[0,1]$ such that:

- For $p<p_{c}$, any infinite-volume measure has no infinite cluster almost surely.

- For $p>p_{c}$, any infinite-volume measure has an infinite cluster almost surely.

PROOF. In order to prove this theorem, simply define $p_{c}:=\inf \left\{p \in[0,1]: \phi_{p, q}^{\text {free }}[0 \longleftrightarrow \infty]>0\right\}=\sup \left\{p \in[0,1]: \phi_{p, q}^{\text {free }}[0 \longleftrightarrow \infty]=0\right\}$.

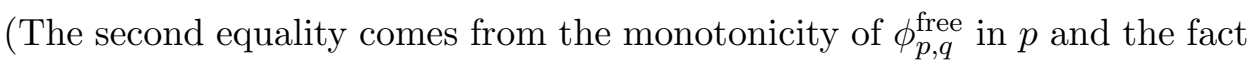
that $0 \longleftrightarrow \infty$ is an increasing event.) For any $p>p_{c}$, the $\phi_{p, q}^{\text {free }}$-probability of having an infinite cluster is 1 , since this event is invariant under translations and the measure $\phi_{p, q}^{\text {free }}$ is ergodic. Therefore, any infinite-volume measure has an infinite cluster almost surely by $(2.3)$. For $p<p_{c}$, choose $p^{\prime} \in\left(p, p_{c}\right)$ for which the infinite-volume measure is unique. In such case, we have that the $\phi_{p^{\prime}, q}^{\text {wired }}$-probability of having an infinite-cluster is 0 since $\phi_{p^{\prime}, q}^{\text {wired }}=\phi_{p^{\prime}, q}^{\text {free }}$. This is true for any $p<p^{\prime}$ by monotonicity, and therefore for any infinite-volume measure since wired boundary conditions are the largest possible.

One may easily check that $p_{c}(q, 1)=1$ for any $q>0$. Also, one may prove that $p_{c}(q, d)>0$. Indeed, for any configuration $\omega$ on edges $f \neq e$, we find that

$$
\phi_{p, q}^{\text {free }}\left[\omega_{e}=1 \mid \omega_{f}: f \neq e\right] \leq p .
$$

This implies by induction that for any set of disjoint edges $e_{1}, \ldots, e_{n}$,

$$
\phi_{p, q}^{\text {free }}\left[\omega_{e_{i}}=1, \forall i \leq n\right] \leq p^{n} .
$$

If 0 is connected to distance $n$, then there must exist a self-avoiding path of adjacent open edges (where adjacent means that for any $0 \leq i<n, e_{i}$ and $e_{i+1}$ share one endpoint). Therefore,

$$
\phi_{p, q}^{\text {free }}\left[0 \longleftrightarrow \partial \Lambda_{n}\right] \leq \sum_{\text {path } e_{1}, \ldots, e_{n}} \phi_{p, q}^{\text {free }}\left[\omega_{e_{i}}=1, \forall i \leq n\right] \leq(2 d p)^{n}
$$

where the sum is over self-avoiding paths of adjacent edges $e_{1}, \ldots, e_{n}$. If $2 d p<1$, we obtain that this quantity tends to 0 as $n$ tends to infinity, thus proving that $p \leq p_{c}(q, d)$. In conclusion, $p_{c}(q, d) \geq 1 /(2 d)$.

2.2.3. A special feature of dimension 2: planarity. While trying to compute $p_{c}(q, d)$ appears very natural to do, a concrete formula is not really expected in general (for instance for $d \geq 3$, the value is probably not rational or algebraic). Nevertheless, dimension two enjoys a particularly nice property, called planar duality, enabling us to derive the critical value rigorously.

Consider the dual of $\mathbb{Z}^{2}$ to be the lattice $\left(\mathbb{Z}^{2}\right)^{*}:=\left(\frac{1}{2}, \frac{1}{2}\right)+\mathbb{Z}^{2}$. By definition, each edge of $\mathbb{Z}^{2}$ crosses exactly one edge of $\left(\mathbb{Z}^{2}\right)^{*}$ which we now denote by $e^{*}$. Any configuration $\omega \in\{0,1\}^{E\left(\mathbb{Z}^{2}\right)}$ corresponds to a dual configuration $\omega^{*} \in\{0,1\}^{E\left(\left(\mathbb{Z}^{2}\right)^{*}\right)}$ via the following relation 


$$
\omega_{e^{*}}^{*}=1-\omega_{e}, \quad \forall e \in E\left(\mathbb{Z}^{2}\right) .
$$

Planar duality refers to the fact that if $\omega$ is sampled according to $\phi_{p, q}^{\text {wired }}$, then $\omega^{*}$ is sampled according to $\phi_{p^{*}, q^{*}}^{\text {free }}\left(\right.$ more precisely a translate by $\left(\frac{1}{2}, \frac{1}{2}\right)$ of the measure, which is therefore defined on $\left.\left(\mathbb{Z}^{2}\right)^{*}\right)$, where

$$
q^{*}=q \quad \text { and } \quad \frac{p p^{*}}{(1-p)\left(1-p^{*}\right)}=q .
$$

Now, fix $q \geq 1$ and observe that $p_{\text {sd }}=p_{\text {sd }}(q):=\sqrt{q} /(1+\sqrt{q})$ is the only value of $p$ such that $p^{*}=p$.

Assume that $p_{c}<p_{\text {sd }}$, in such case there exists an infinite cluster of open edges at $p_{\mathrm{sd}}$. But the dual model of $\phi_{p_{\mathrm{sd}}, q}^{\text {free }}$ is a translate by $\left(\frac{1}{2}, \frac{1}{2}\right)$ of $\phi_{p_{\mathrm{sd}}, q}^{\text {wired }}$, and therefore there exists an infinite cluster of dual-open edges on $\left(\mathbb{Z}^{2}\right)^{*}$ as well. In other words, there is coexistence of an infinite cluster in $\omega$ and an infinite cluster in $\omega^{*}$. This fact can appear counter-intuitive physically, and can indeed be ruled out mathematically. This sketch of argument therefore suggests that $p_{c} \geq p_{\text {sd }}$.

Now assume that $p_{c}>p_{\text {sd }}$. Then there is no infinite cluster in $\omega$ or $\omega^{*}$ for any $p \in\left(p_{\mathrm{sd}}, p_{c}\right)$. This fact can be proved to be occurring at one value of $p$ maximum (which turns out to be $p_{\mathrm{sd}}$ ), and is the main object of the following theorem.

Theorem 2.6 (Beffara, DC [BD12a, DRT15]). For $q \geq 1$, the critical value $p_{c}=p_{c}(q, 2)$ satisfies

$$
p_{c}=\frac{\sqrt{q}}{1+\sqrt{q}} .
$$

One may easily check that $p_{c}(q, d)$ is decreasing in $d$. Therefore, $p_{c}(q, d) \leq$ $\frac{\sqrt{q}}{1+\sqrt{q}}$ for $d \geq 2$, thus showing that $p_{c}(q, d)<1$.

2.2.4. Phase transition in the Potts model. Corollary 2.4 implies that

$$
\lim _{n \rightarrow \infty} \mu_{\Lambda_{n}, \beta, q}^{1}\left[\sigma_{0}\right]=\lim _{n \rightarrow \infty} \phi_{\Lambda_{n}, p, q}^{\mathrm{wired}}[0 \leftrightarrow \infty] \cdot \overrightarrow{1}=\phi_{p, q}^{\mathrm{wired}}[0 \leftrightarrow \infty] \cdot \overrightarrow{1},
$$

where $\beta=-\frac{q-1}{q} \log [1-p]$. We deduce the following result:

TheOREM 2.7. The quantity $\beta_{c}=\beta_{c}(q, d):=-\frac{q-1}{q} \log \left[1-p_{c}(q, d)\right] \in$ $(0, \infty)$ satisfies

$$
\lim _{n \rightarrow \infty} \mu_{\Lambda_{n}, \beta, q}^{1}\left[\sigma_{0}\right] \quad \begin{cases}=0 & \text { if } \beta<\beta_{c} \\ \neq 0 & \text { if } \beta>\beta_{c} .\end{cases}
$$

Furthermore, we have that

$$
m^{*}(\beta, q):=\lim _{n \rightarrow \infty} m\left(\Lambda_{n}, \beta, q\right)=\lim _{n \rightarrow \infty} \mu_{\Lambda_{n}, \beta, q}^{1}\left[\sigma_{0}\right] .
$$

The first claim is due to the coupling between FK percolation and the Potts model. The second claim follows from the fact that the mean of the 
spins averaged on the whole box $\Lambda_{n}$ converges as $n$ tends to infinity to the mean of the spin at the origin. This can easily be checked using the ergodicity of $\phi_{p, q}^{\text {wired }}$, we omit this (fairly easy) proof here.

In words, the previous theorem states that the critical inverse temperature of the Potts model can be defined rigorously (it does not alternate between ordered and disordered phases as it a priori could), and this critical inverse-temperature can be expressed in terms of the critical value of the corresponding FK percolation. In two dimensions, we immediately obtain that $\beta_{c}(q, 2)=\frac{q-1}{q} \log (1+\sqrt{q})$ thanks to Theorem 2.6.

Also note the icing on the cake: the phase transition of the Potts model is continuous if and only if $\phi_{p_{c}, q}^{\text {wired }}[0 \leftrightarrow \infty]=0$. As a consequence, we can focus our attention on FK percolation in order to determine whether the phase transition is continuous or discontinuous. We do so in the next section.

\section{Critical behavior on $\mathbb{Z}^{2}$}

In this section, we focus on the two dimensional case. For now on, we work with FK percolation, and only briefly mention the consequences for the Potts model.

3.1. An alternative between two possible critical behaviors. Physicists possess several definitions of continuous phase transitions. For instance, it may refer to the divergence of the correlation length, the continuity of the order parameter (here the spontaneous magnetization), the uniqueness of the Gibbs states at criticality, the divergence of the susceptibility, the scale invariance at criticality, etc. From a mathematical point of view, these properties are not clearly equivalent (there are examples of models for which they are not), and they therefore refer to a priori different notions of continuous phase transition. The following result shows that all these properties are equivalent for the planar FK percolation (and therefore for the associated Potts model). As a consequence, the previous properties are alternative characterizations of a single notion, and we may think of the notion of a continuous phase transition.

Theorem 3.1 (DC-Sidoravicius-Tassion [DST15]). Let $q \geq 1$, the following assertions are equivalent at criticality:

$\mathbf{P 1}$ (Absence of infinite cluster) $\phi_{p_{c}, q}^{\text {wired }}[0 \longleftrightarrow \infty]=0$.

$\mathbf{P 2}$ (Uniqueness of the infinite-volume measure) $\phi_{p_{c}, q}^{\mathrm{free}}=\phi_{p_{c}, q}^{\text {wired }}$.

P3 (Infinite susceptibility) $\sum_{x \in \mathbb{Z}^{2}} \phi_{p_{c}, q}^{\text {free }}[0 \longleftrightarrow x]=\infty$.

P4 (Sub-exponential decay of correlations for free boundary conditions)

$$
\lim _{n \rightarrow \infty} \frac{1}{n} \log \phi_{p_{c}, q}^{\text {free }}\left[0 \longleftrightarrow \partial \Lambda_{n}\right]=0 .
$$

P5 $(R S W)$ Let $\alpha>0$. There exists $c=c(\alpha)>0$ such that for all $n \geq 1$ and any boundary conditions $\xi$, 


$$
c \leq \phi_{[-n,(\alpha+1) n] \times[-n, 2 n], p_{c}, q}^{\xi}\left[\begin{array}{l}
{[0, \alpha n] \times[0, n] \text { is crossed from }} \\
\text { left to right by an open path }
\end{array}\right] \leq 1-c .
$$

The previous theorem does not show that these conditions are all satisfied, only that they are equivalent. In fact, whether the conditions are satisfied or not depend on the value of $q$, as we will see later in this review.

Property P5 is the strongest one. Note that we did not only require that the probability of being crossed by an open path remains bounded away from 0 uniformly in the size $n$ of the rectangle, but also uniformly in boundary conditions. This property is crucial for the applications of this theorem, in particular since it helps controlling the dependencies between edges in different parts of the graph. Among other results, P5 implies

- the polynomial decay of correlations at criticality,

- the existence of sub-sequential scaling limits,

- the value for certain critical exponents called universal critical exponents (it has nothing to do with the universality for the model itself),

- the fractal nature of large clusters (with some explicit bounds on the Hausdorff dimension).

Furthermore, it represents an important tool for the following problems:

- understanding the conformal invariance of the model,

- understanding scaling relations between several critical exponents,

- proving the universal behavior at criticality.

This result was previously known in a few cases. For $q=1$, the properties follow from a collection of results, among which the two fundamental papers of Russo [Rus78] and Seymour and Welsh [SW78]. For $q \gg 1$, the above properties fail (see $\left[\mathbf{K S 8 2}, \mathbf{L M M S}^{+} \mathbf{9 1}\right]$ ), while for $q=2$ (which is coupled to the Ising model), all of these properties are proved to be true using specific properties of the Ising model; see [Ons44, Sim80, DHN11].

Before proceeding forward, let us discuss very briefly the ingredients in the proof for other values of $q \geq 1$. As mentioned before, $\mathbf{P} 5$ is the strongest property. On the other hand, $\mathbf{P} 4$ is the weakest. Therefore, it does not come as a surprise that $\mathbf{P 5} \Rightarrow \mathbf{P} \mathbf{1} \Rightarrow \mathbf{P} \mathbf{2} \Rightarrow \mathbf{P 3} \Rightarrow \mathbf{P} 4$ is fairly easy to obtain. The main difficulty lies in the proof of $\mathbf{P} \mathbf{4} \Rightarrow \mathbf{P 5}$, or equivalently that

$$
\inf \left\{\phi_{[-n,(\alpha+1) n] \times[-n, 2 n], p_{c}, q}^{\text {free }}\left[\begin{array}{l}
{[0, \alpha n] \times[0, n] \text { is crossed from }} \\
\text { left to right by an open path }
\end{array}\right] \quad, \quad n \geq 1\right\}=0
$$

implies that the probability of being connected to distance $n$ tends to 0 exponentially fast (i.e. nonP4). (Since the free boundary conditions are the smallest, and since the uniform upper bound on crossing probabilities can be obtained via duality and the uniform lower bound, (3.1) is equivalent to nonP5.)

In order to prove this implication, we developed a new geometric renormalization principle for crossing probabilities: crossing probabilities at scale $2 n$ are expressed in terms of crossing probabilities at scale $n$. The renormal- 
ization scheme is built in such a way that as soon as the crossing probability passes below a certain threshold, crossing probabilities start decaying exponentially fast. As a consequence, either crossing probabilities remain bounded away from 0 , or they decay to 0 exponentially fast.

The major difficulty arising in the implementation of this renormalization scheme is to handle long-range dependency and boundary conditions properly. The lack of independence renders the proof difficult, and requires the introduction of completely new tools. Let us finish by mentioning that historically, Kesten showed $\mathbf{P} \mathbf{4} \Rightarrow \mathbf{P 5}$ for Bernoulli percolation but his proof cannot be extended to FK percolation with $q>1$.

3.2. Deciding between critical behaviors: the loop representation and Baxter's conjecture. We should now decide whether, for a fixed $q \geq 1$, properties $\mathbf{P} \mathbf{1}-\mathbf{5}$ are satisfied or not. In order to do so, we introduce yet another representation of the Potts model. It is derived directly from the planar FK percolation. We start by defining the loop configuration $\bar{\omega}$ associated to a percolation configuration $\omega$. Let $\left(\mathbb{Z}^{2}\right)^{\diamond}$ be the lattice defined as follows. The set of vertices is given by the midpoints of edges of $\mathbb{Z}^{2}$. The edges are all the pairs of nearest vertices (i.e. vertices at distance $\sqrt{2} / 2$ of each others). It is a rotated and rescaled version of the square lattice, see Fig. 6. For future reference, note that the edges of the medial lattice can be oriented in a counter-clockwise way around faces that are centered on a vertex of $\mathbb{Z}^{2}$.

Let $\Omega$ be a finite subgraph of $\mathbb{Z}^{2}$. Let $\Omega^{*}$ be the subgraph of $\left(\mathbb{Z}^{2}\right)^{*}$ induced by dual edges bordering faces of $\left(\mathbb{Z}^{2}\right)^{*}$ corresponding to vertices of $\Omega$. Let $\Omega^{\diamond}$ be the subgraph of $\left(\mathbb{Z}^{2}\right)^{\diamond}$ defined by the vertices corresponding to midpoints of edges in $E(\Omega)$, and edges between two vertices of $V\left(\Omega^{\diamond}\right)$ on the same face of $\Omega$.

Consider a configuration $\omega$ together with its dual configuration $\omega^{*}$ (recall that $\left.\omega_{e^{*}}^{*}=1-\omega_{e}\right)$. We draw $\omega^{*}$ in such a way that dual edges between vertices of $\partial \Omega^{*}$ are open in $\omega^{*}$ (we make such an arbitrary choice since these dual edges have no corresponding edges in $E(\Omega)$ ).

By definition, through every vertex of the medial graph $\Omega^{\diamond}$ of $\Omega$ passes either an edge of $\omega$ or an edge of $\omega^{*}$. Draw self-avoiding loops on $\Omega^{\diamond}$ as follows: a loop arriving at a vertex of the medial lattice always makes a $\pm \pi / 2$ turn at vertices so as not to cross the edges of $\omega$ or $\omega^{*}$, see Fig. 7 . The loop configuration is defined in an unequivocal way since:

- there is either an edge of $\omega$ or an edge of $\omega^{*}$ crossing non-boundary vertices in $\Omega^{\diamond}$, and therefore there is exactly one coherent way for the loop to turn at non-boundary vertices.

- there is only one possible $\pm \pi / 2$ turn at boundary vertices keeping the loops in $\Omega^{\diamond}$.

From now on, the loop configuration associated to $\omega$ is denoted by $\bar{\omega}$.

We allow ourselves a slight abuse of notation: below, $\phi_{\Omega, p, q}^{0}$ denotes the measure on percolation configurations as well as its push-forward by the map 


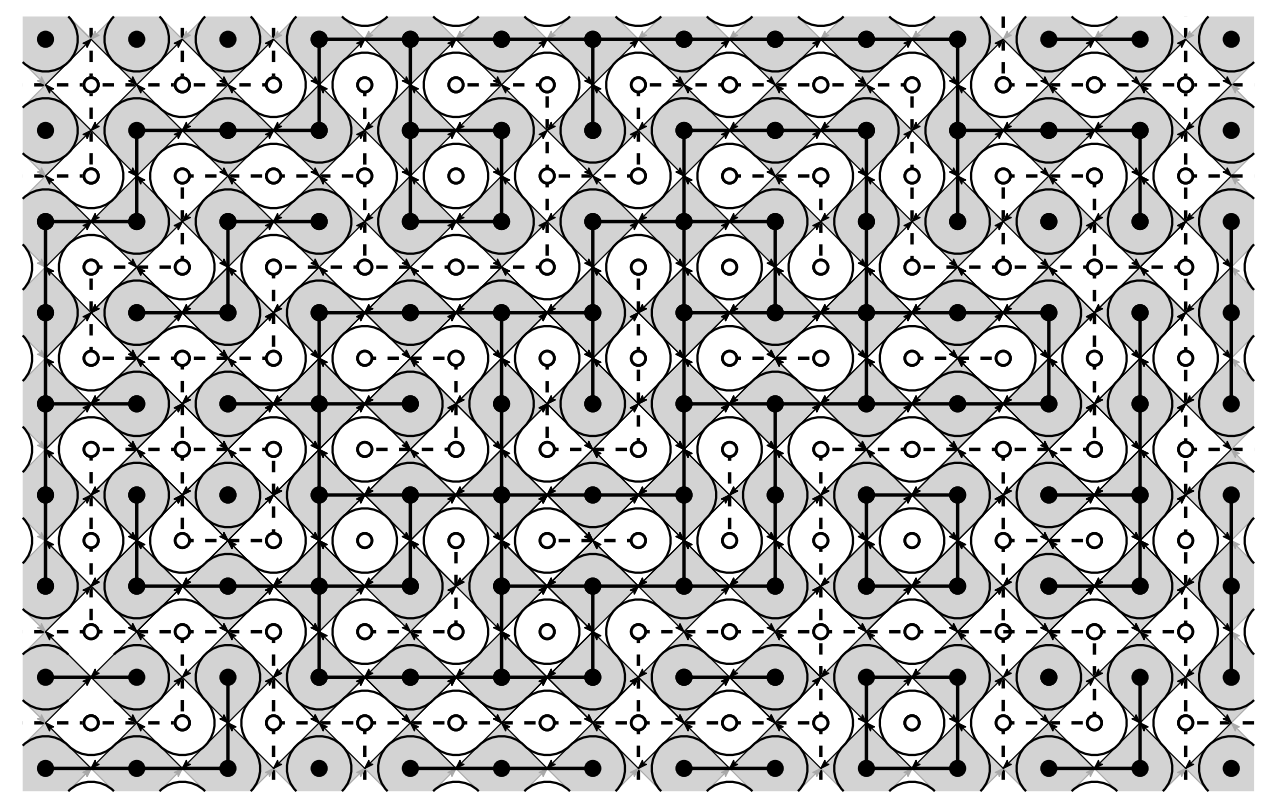

Figure 7. The configurations $\omega$ (in bold lines), $\omega^{*}$ (in dashed lines) and $\bar{\omega}$ (in plain lines).

$\omega \mapsto \bar{\omega}$. Therefore, the measure $\phi_{\Omega, p, q}^{0}$ will sometimes refer to a measure on loop configurations. Fix

$$
x=x(p, q):=\frac{p}{\sqrt{q}(1-p)} .
$$

Proposition 3.2. Let $\Omega$ be a connected finite subgraph of $\mathbb{Z}^{2}$ which complement in $\mathbb{Z}^{2}$ is connected. Let $p \in[0,1]$ and $q>0$. For any configuration $\omega$,

$$
\phi_{\Omega, p, q}^{\text {free }}[\bar{\omega}]=\frac{x^{o(\omega)} \sqrt{q}^{\ell(\bar{\omega})}}{Z(\Omega, p, q)},
$$

where $\ell(\bar{\omega})$ is the number of loops in $\bar{\omega}$ and $Z(\Omega, p, q)$ is a normalizing constant.

In particular, when $p=p_{c}(q)$, we obtain that $x=1$ and the probability of a loop configuration is expressed in terms of the number of loops only.

Proof. An induction on the number of open edges shows that

$$
\ell(\bar{\omega})=2 k(\omega)+o(\omega)-|V(\Omega)| .
$$

Indeed, if there is no open edge, then $\ell(\bar{\omega})=k(\omega)=|V(\Omega)|$. Now, adding an edge can either:

- join two connected components of $\omega$, thus decreasing the number of loops and the number of connected components by 1 ,

- close a cycle in $\omega$, thus increasing the number of loops by 1 and not changing the number of connected components. 


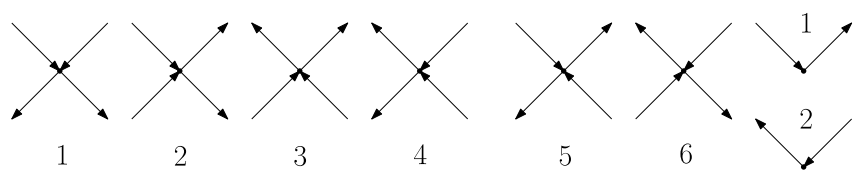

FiguRE 8. Different possible patterns for the six vertex model on the medial lattice. The two patterns on the right are boundary patterns.

Equation (3.2) implies that

$$
\begin{aligned}
p^{o(\omega)} & (1-p)^{c(\omega)} q^{k_{\text {free }}(\omega)} \\
= & p^{o(\omega)}(1-p)^{|E(\Omega)|-o(\omega)} q^{k_{\text {free }}(\omega)} \\
= & (1-p)^{|E(\Omega)|} \sqrt{q}^{|V(\Omega)|}\left(\frac{p}{(1-p) \sqrt{q}}\right)^{o(\omega)} \sqrt{q}^{2 k_{\text {free }}(\omega)+o(\omega)-|V(\Omega)|} \\
= & (1-p)^{|E(\Omega)|} \sqrt{q}^{|V(\Omega)|} x^{o(\omega)} \sqrt{q}^{\ell(\bar{\omega})} .
\end{aligned}
$$

The proof follows by setting

$$
\widehat{Z}(\Omega, p, q):=\frac{Z(\Omega, p, q)}{(1-p)^{|E(\Omega)|} \sqrt{q}^{|V(\Omega)|}} .
$$

The loop representation of the FK percolation is a well-known representation. It allows to map the free energies of the Potts model and the FK percolation to the free energy of a solid-on-solid ice-type model. For completeness, we succinctly present the mapping here and we refer to [Bax89, Chapter 10] for more details on the subject. We focus on the case of a domain $\Omega$ with free boundary conditions.

From a loop configuration $\bar{\omega}$, one may deduce a configuration $\vec{\omega}$ of arrows on edges as follows. Orientate loops of $\bar{\omega}$ clockwise or counterclockwise. Then, forget the way loops turn at every vertex (simply remember which orientation the loops give to each edges of the medial graph). This procedure gives rise to a configuration $\vec{\omega}$ of arrows on edges of $\Omega^{\diamond}$ with the constraint that for every vertex of $\Omega^{\diamond}$, the number of incoming arrows is equal to the number of outgoing arrows. The set of possible configurations is denoted by $\mathrm{A}(\Omega)$.

Assume for a moment that we consider directly a model, called the 6 vertex model, on $A(\Omega)$ for which the probability of $\vec{\omega}$ is given by

$$
\mathrm{P}[\vec{\omega}]=\frac{x_{1}^{N_{1}(\vec{\omega})} x_{2}^{N_{2}(\vec{\omega})} x_{3}^{N_{3}(\vec{\omega})} x_{4}^{N_{4}(\vec{\omega})} x_{5}^{N_{5}(\vec{\omega})} x_{6}^{N_{6}(\vec{\omega})} y_{1}^{\tilde{N}_{1}(\vec{\omega})} y_{2}^{\tilde{N}_{2}(\vec{\omega})}}{Z_{6 V}\left(x_{1}, x_{2}, x_{3}, x_{4}, x_{5}, x_{6}, y_{1}, y_{2}\right)}
$$

where $x_{1}, \ldots, x_{6}, y_{1}, y_{2}>0$, and $N_{1}, \ldots, N_{6}, \widetilde{N}_{1}, \widetilde{N}_{2}$ correspond to the number of vertices with local arrangements of arrows of types 1-6 and boundary vertices with local arrangements of arrows of types 1-2; see Fig. 8. 
We may consider the free energy of this model, defined by

$$
f_{6 \mathrm{~V}}\left(x_{1}, \ldots, x_{6}\right):=\lim _{n \rightarrow \infty} \frac{1}{\left|E\left(\Lambda_{n}\right)\right|} \log Z_{6 \mathrm{~V}}\left(\Lambda_{n}, x_{1}, \ldots, x_{6}, y_{1}, y_{2}\right) .
$$

(We do not recall the dependency in $y_{1}$ and $y_{2}$ since the quantity can easily be seen to be independent of $y_{1}$ and $y_{2}$ in the limit $n \rightarrow \infty$.)

We already know from the coupling between the Potts model and FK percolation that the partitions functions of the two models are related. We also know that at criticality, the partition function of FK percolation is related to the partition function of the loop model. The transformation between the loop model and the 6 vertex model mentioned briefly above can be used to prove the following provided that the weight of a configuration of oriented loops obtained from $\bar{\omega}$ is proportional to the probability of $\bar{\omega}$ times $e^{2 \pi \mathrm{i} \tilde{\sigma}}$ to the number of loops oriented counterclockwise times $e^{-2 \pi \mathrm{i} \tilde{\sigma}}$ to the number of loops oriented clockwise, where $\tilde{\sigma}$ is chosen carefully.

Proposition 3.3 (Baxter [Bax89]). Let $G$ be a finite graph, we have

$$
f_{\text {Potts }}\left(\beta_{c}, q\right)=\frac{2 \beta_{c}}{q-1}-\log q+2 f_{6 \mathrm{~V}}(1,1,1,1,2 \cos (\pi \tilde{\sigma}), 2 \cos (\pi \tilde{\sigma}))
$$

where $\cos (2 \pi \tilde{\sigma})=\sqrt{q} / 2$.

The main advantage of the 6 vertex model over the Potts model is that it is exactly solvable. In other words, one may compute the free energy of the model via transfer matrices and the so-called Bethe Ansatz (see [Bax89] and references therein). As a result, the free energy of the critical Potts model can be computed explicitly. Note that this provides little information on the critical behavior of the Potts model since thermodynamical quantities of the model are expressed in terms of derivatives of the free energy. Computing the free energy at one point only is not sufficient to access these derivatives. However, Baxter [Bax71, Bax73, Bax78, Bax89] used this correspondence together with additional unproved assumptions to state the following conjecture.

Conjecture 2. Consider the Potts model on the square lattice. For $q \leq 4$, the phase transition is continuous (and therefore $\mathbf{P} \mathbf{1}-\mathbf{5}$ are satisfied), while for $q>4$ it is discontinuous (thus $\mathbf{P} \mathbf{1}-\mathbf{5}$ are not satisfied).

In [LMR86, $\mathbf{L M M S}^{+} \mathbf{9 1}, \mathbf{K S 8 2}$ ], the FK percolation was proved to undergo a discontinuous phase transition at criticality when $q \geq 25.72$ via a Pirogov-Sinai type argument, see also the proof in [Dum15]. In the next section, we focus on the regime of values of $q$ for which the phase transition is continuous, i.e. $q \in[1,4]$. This leaves the range of parameter $q \in(4,25.72)$ open to analysis (in such case one should prove that the phase transition is discontinuous). 
3.3. Values of $q$ for which the phase transition is continuous. In this section, we focus on the $q \leq 4$ case. We shall discuss the following theorem.

Theorem 3.4 (Duminil-Copin [Dum12]). Let $1 \leq q \leq 4$, then

$$
\lim _{n \rightarrow \infty} \frac{1}{n} \log \phi_{p_{c}, q}^{0}\left[0 \longleftrightarrow \partial \Lambda_{n}\right]=0 .
$$

This theorem, combined with Theorem 3.1, implies the following corollary.

Corollary 3.5. The phase transition is continuous for the FK percolation with $1 \leq q \leq 4$, and therefore for the Potts models with 2, 3 and 4 colors.

The case of the Ising model (i.e. $q=2$ ) was solved by Onsager [Ons44].

3.3.1. Definition of parafermionic observables. In order to prove this result, we introduce a new tool called parafermionic observable. This observable is easier to understand in the context of Dobrushin boundary conditions, which are defined as follows. The following paragraphs are difficult to read, and Fig. 9 may replace conveniently the tedious definitions below.

Let $\Omega$ be a connected graph with connected complement in $\mathbb{Z}^{2}$, and $a$ and $b$ two vertices on its boundary. The triplet $(\Omega, a, b)$ is called a Dobrushin domain. The set $\partial \Omega$ is divided into two boundary arcs denoted by $\partial_{a b}$ and $\partial_{b a}$ (the first one goes from $a$ to $b$ when going counterclockwise around $\partial \Omega$, while the second goes from $b$ to $a$ ). The Dobrushin boundary conditions are

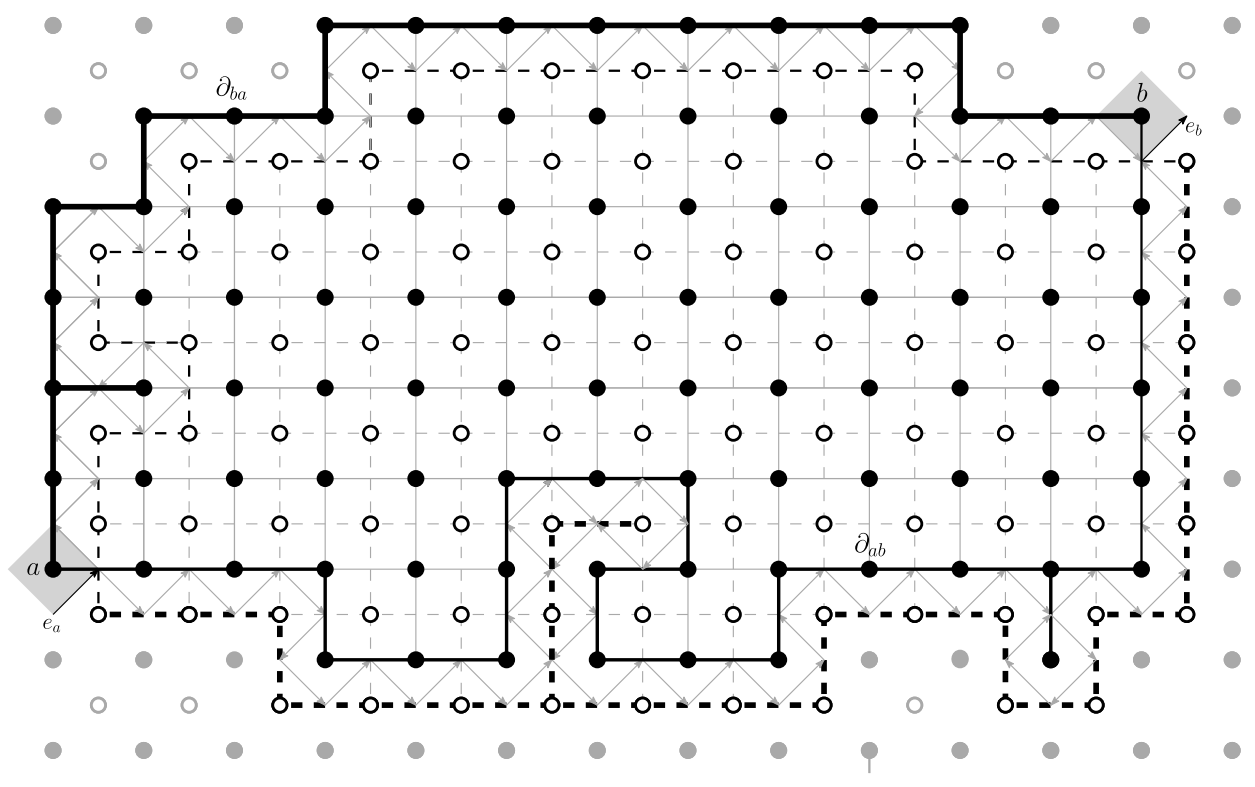

Figure 9. The primal and dual Dobrushin domains associated to a medial Dobrushin domain. Note the position of $a$ and $b$ and the definition of $\partial_{b a}$ and $\partial_{a b}$. 


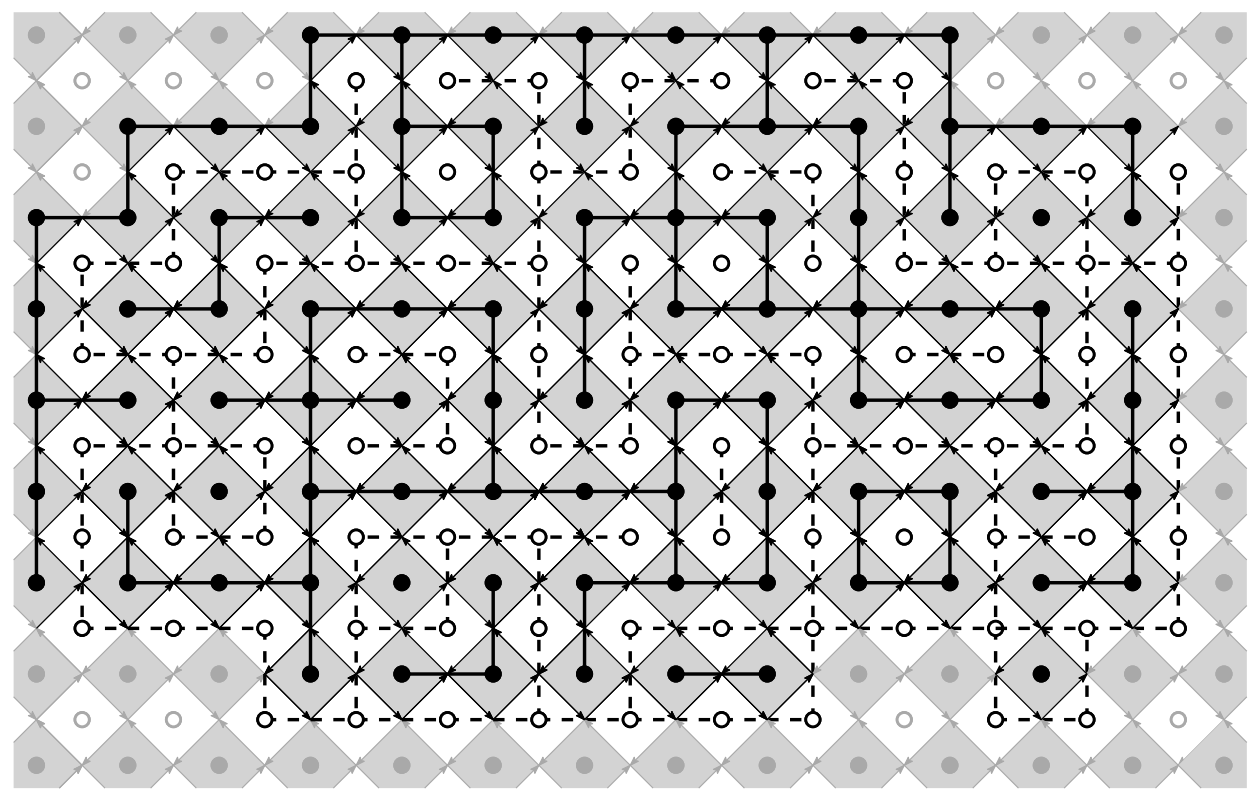

Figure 10. The configuration $\omega$ with its dual configuration $\omega^{*}$. Notice that the edges of $\omega$ are open on $\partial_{b a}$, and that those of $\omega^{*}$ are open on the part of the boundary of $\Omega^{*}$ bordering $\partial_{a b}$.

defined to be free on $\partial_{a b}$ and wired on $\partial_{b a}$. In other words, the partition is composed of $\partial_{b a}$ together with singletons.

Let $\Omega^{*}$ be the dual of the graph $\Omega$ whose set of edges is given by the edges of $\left(\mathbb{Z}^{2}\right)^{*}$ crossing an edge of $E(\Omega) \backslash \partial_{b a}$ and set of vertices given by the endpoints of these edges, see Fig. 9. Draw $\omega$ with the additional condition that edges of $\partial_{b a}$ are open, and the dual $\omega^{*}$ with the additional condition that edges on $\partial \Omega^{*}$ bordering $\partial_{a b}$ are open in $\omega^{*}$.

Define the medial graph $\Omega^{\diamond}$ of $\Omega$ as follows: the vertices are the vertices of $\left(\mathbb{Z}^{2}\right)^{\diamond}$ at the center of edges of $\Omega$ or $\Omega^{*}$. Let $e_{a}$ and $e_{b}$ be the two medial edges defined as on Fig. 9.

One may define a loop configuration $\bar{\omega}$ exactly as before, which this time contains loops together with a self-avoiding path going from $e_{a}$ to $e_{b}$, see Figures 9-11. This curve is called the exploration path and is denoted by $\gamma=\gamma(\omega)$. The loops correspond to the interfaces separating clusters from dual clusters, and the exploration path corresponds to the interface between the vertices connected in $\omega$ to $\partial_{b a}$ and the dual vertices connected in $\omega^{*}$ to $\partial_{a b}^{*}$.

The following definition will be instrumental in the reminder of this section.

Definition 3.6. The winding $W_{\Gamma}\left(e, e^{\prime}\right)$ of a curve $\Gamma$ (on the medial lattice) between two medial-edges $e$ and $e^{\prime}$ of the medial graph is the total 


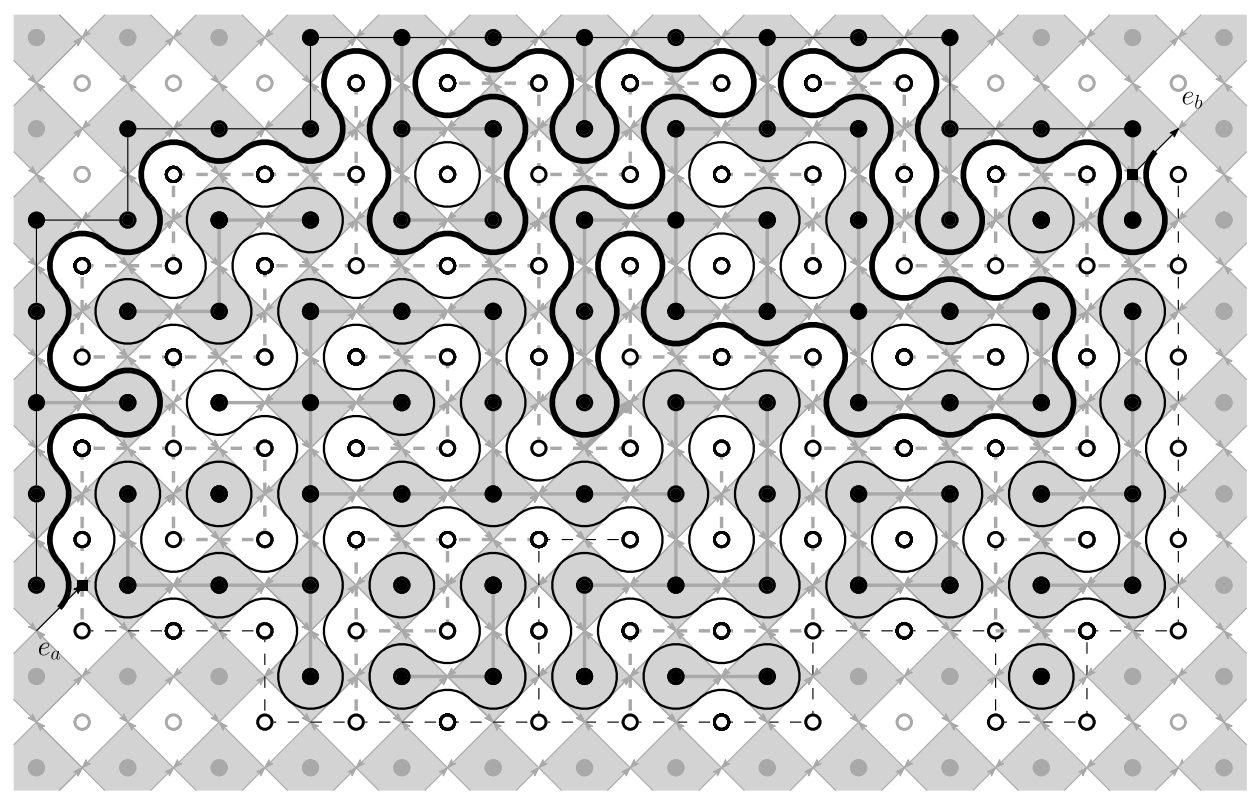

Figure 11 . The loop configuration $\bar{\omega}$ associated to the primal and dual configurations $\omega$ and $\omega^{*}$ in the previous picture. The exploration path is drawn in bold. It starts at $e_{a}$ and finishes at $e_{b}$.

signed rotation in radians that the (now oriented) curve makes from the mid-point of the edge e to that of the edge $e^{\prime}$. By convention, if $\Gamma$ does not go through $e^{\prime}$, we set $W_{\Gamma}\left(e, e^{\prime}\right)=0$.

The winding of $\Gamma$ can be computed in a very simple way: it corresponds to the number of $\frac{\pi}{2}$-turns on the left minus the number of $\frac{\pi}{2}$-turns on the right times $\pi / 2$.

We are now in a position to define the parafermionic observable.

Definition 3.7. Consider a Dobrushin domain $(\Omega, a, b)$. The parafermionic observable $F=F(\Omega, p, q, a, b)$ is defined for any medial edge $e \in$ $E\left(\Omega^{\diamond}\right)$ by

$$
F(e):=\phi_{\Omega, p, q}^{\text {dobr }}\left[\mathrm{e}^{\mathrm{i} \sigma W_{\gamma}\left(e, e_{b}\right)} \mathbf{1}_{e \in \gamma}\right],
$$

where $\phi_{\Omega, p, q}^{\text {dobr }}$ is the measure with Dobrushin boundary conditions on $(\Omega, a, b)$ and $\gamma$ is the exploration path and $\sigma$ is a solution of the equation

$$
\sin (\sigma \pi / 2)=\sqrt{q} / 2 .
$$

Note that $\sigma$ belongs to $\mathbb{R}$ for $q \leq 4$ and to $1+i \mathbb{R}$ for $q>4$. This hints that the critical behavior of FK percolation is different for $q>4$ and $q \leq 4$. For $q \in[0,4], \sigma$ has the physical interpretation of a spin, which is fractional in general, hence the name parafermionic (fermions have half-integer spins while bosons have integer spins, there are no particles with fractional spin, but the use of such fractional spins at a theoretical level has been very 
fruitful in physics). For $q>4, \sigma$ is not real anymore and does not have any physical interpretation.

These observables first appeared in the context of the Ising model (there they are called order-disorder operators) and dimer models. They were later on extended to FK percolation and the loop $O(n)$-model by Smirnov [Smi06] (see [DS12a] for more details). Since then, these observables have been at the heart of the study of these models. For an example of applications in a different context (for which the observable is simple to define and may appeal more to the reader), we refer to $\left[\mathbf{D S 1 2 b}, \mathbf{B B M d G}^{+} \mathbf{1 4}, \mathbf{G l a 1 3}\right]$ for a study of self-avoiding walks.

3.3.2. Contour integrals of the parafermionic observable. The parafermionic observable satisfies a spectacular property at criticality.

Let $(\Omega, a, b)$ be a Dobrushin domain. A discrete contour $\mathscr{C}$ is a finite sequence $z_{0} \sim z_{1} \sim \cdots \sim z_{n}=z_{0}$ in $V(\Omega) \cup V\left(\Omega^{*}\right)$ of neighboring points (meaning that they are at the center of adjacent faces of $\Omega^{\diamond}$ ) such that the path $\left(z_{0}, \ldots, z_{n}\right)$ is edge-avoiding. The discrete contour integral of the parafermionic observable $F$ along $\mathscr{C}$ is defined by

$$
\oint_{\mathscr{C}} F(z) d z:=\sum_{i=0}^{n-1}\left(z_{i+1}-z_{i}\right) F\left(\left\{z_{i}, z_{i+1}\right\}^{*}\right),
$$

where the $z_{i}$ are considered as complex numbers and $\left\{z_{i}, z_{i+1}\right\}^{*}$ denotes the edge of $\Omega^{\diamond}$ intersecting $\left\{z_{i}, z_{i+1}\right\}$ in its center.

TheOREm 3.8 (Vanishing contour integrals). Let $q>0, p=p_{c}$, and $a$ Dobrushin domain $(\Omega, a, b)$. For any discrete contour $\mathscr{C}$ of $(\Omega, a, b)$,

$$
\oint_{\mathscr{C}} F(z) d z=0 .
$$

The property that discrete contour integrals vanish seems to correspond to a well-known property of holomorphic functions: their contour integrals are equal to 0 . Nevertheless, one should be slightly careful when drawing such a parallel: the observable should rather be understood as the discretization of a form rather than a function. As a form, the fact that these discrete contour integrals vanish should be interpreted as the discretization of the property of being closed.

A troubling news is the fact that this property does not determine the function $F$ (for instance, even if boundary conditions are given, there is more than one function $F$ satisfying these boundary conditions and having zero contour integrals). Therefore, it is a priori not clear how one should be able to extract information from this property, yet the following section illustrates the fact that it can indeed be done.

3.3.3. Sketch of the proof of Theorem 3.4. Let us exploit the fact that the discrete integral along the boundary of a domain equals 0 . Let $\mathbb{H}:=\mathbb{N} \times \mathbb{Z}$. Taking the domain to be the rectangle $U_{n}:=\Lambda_{n} \cap \mathbb{H}$, (3.4) applied to the discrete contour going around $U_{n}$ (i.e the unique contour crossing each edge 
of $U_{n}^{\diamond}$ incident to exactly one vertex of $\partial U_{n}^{\diamond}$ ) implies (after some work that we conveniently avoid presenting here) the following inequality: for $q \leq 2$, there exists $c=c(q)>0$ such that for any $n \geq 1$,

$$
\sum_{x \in \partial \Lambda_{n}} \phi_{U_{n}, p_{c}, q}^{\text {free }}[0 \leftrightarrow x] \geq c .
$$

Note that $\phi_{U_{n}, p_{c}, q}^{\text {free }}[0 \leftrightarrow x]$ is non zero for $x \in \partial \Lambda_{n}$ only if $x$ is also on $\partial U_{n}$. Therefore, the previous sum corresponds to the sum over vertices on the boundary of $U_{n}$ that are exactly at distance $n$ from the origin.

Using the comparison between boundary conditions, this implies that

$$
\sum_{x \in \mathbb{Z}^{2}} \phi_{p_{c}, q}^{\text {free }}[0 \leftrightarrow x] \geq \sum_{n \geq 1} \sum_{x \in \partial \Lambda_{n}} \phi_{U_{n}, p_{c}, q}^{\text {free }}[0 \leftrightarrow x]=\infty .
$$

The divergence of this series implies Theorem 3.4 for $q \leq 2$.

The following discussion motivates why the case $q \in[2,4]$ is more difficult. We do not provide much details but rather try to convey an important idea.

It is natural to predict that the following quantity decays like a power law:

$$
\phi_{\mathbb{H}, p_{c}, q}^{\mathrm{free}}\left[0 \longleftrightarrow \partial \Lambda_{n / 2}\right]=\frac{1}{n^{\alpha(q, \pi)+o(1)}},
$$

where $\alpha(q, \pi)$ is a constant depending on $q$ only ( $\pi$ refers to the "angle of the opening of $\mathbb{H}$ " at 0 ), and $o(1)$ denotes a quantity tending to 0 as $n$ tends to infinity.

Moreover, one may argue (it is not straightforward) that the event that $x$ is connected to 0 in $U_{n}$ has a probability close to the probability that 0 and $x$ are connected to distance $n / 2$ in $U_{n}$. For $x$ not too close to the corners of the rectangle, the boundary of $U_{n}$ looks like a straight line and it is therefore natural to predict that

$$
\phi_{U_{n}, p_{c}, q}^{\text {free }}[0 \leftrightarrow x]=\frac{1}{n^{2 \alpha(q, \pi)+o(1)}} .
$$

Summing over all $x$ (for ease of exposition, let us ignore the problem of vertices at the corners), we deduce that

$$
\sum_{x \in \partial \Lambda_{n}} \phi_{U_{n}, p_{c}, q}^{\mathrm{free}}[0 \leftrightarrow x]=n^{1-2 \alpha(q, \pi)+o(1)} .
$$

Now, it is conjectured in physics that

$$
\alpha(q, \pi)=1-2 \frac{\arccos (\sqrt{q} / 2)}{\pi} .
$$

Therefore for $q \in(2,4]$, the quantity on the left-hand side of $(3.7)$ is converging to 0 as $n \rightarrow \infty$ and it is therefore hopeless to get (3.5) for $q>2$.

Nevertheless, we did not have to consider $\mathbb{H}$ in the first place. For instance, consider the graph $\mathbb{S}$ obtained by taking $\mathbb{Z}^{2}$ minus the half-line 
$\{(-n, 0), n \geq 0\}$. As before, one expects that

$$
\phi_{\mathbb{S}, p_{c}, q}^{\text {free }}\left[0 \longleftrightarrow \partial \Lambda_{n / 2}\right]=\frac{1}{n^{\alpha(q, 2 \pi)+o(1)}},
$$

where $\alpha(q, 2 \pi)$ is a value which is a priori smaller than $\alpha(q, \pi)$ since $\mathbb{S}$ is larger $(2 \pi$ refers this time to the "opening angle of $\mathbb{S}$ at" 0$)$.

Therefore, if one applies the same reasoning as above to $\widetilde{U}_{n}:=\Lambda_{n} \cap \mathbb{S}$, we may prove that

$$
\sum_{x \in \partial \Lambda_{n}} \phi_{\widetilde{U}_{n}, p_{c}, q}^{\text {free }}[0 \leftrightarrow x]=n^{1-\alpha(q, \pi)-\alpha(q, 2 \pi)+o(1)} .
$$

(We use that for $x \in \partial \Lambda_{n}$ not close to corners, the boundary looks straight, and that the boundary in $\widetilde{U}_{n}$ near 0 looks like in $\mathbb{S}$.) Since the map $z \mapsto z^{2}$ maps $\mathbb{R}_{+}^{*} \times \mathbb{R}$ to $\mathbb{R}^{2} \backslash-\mathbb{R}_{+}$, conformal invariance predicts that $\alpha(q, 2 \pi)=$ $\alpha(q, \pi) / 2$. As a consequence,

$$
\sum_{x \in \partial \Lambda_{n}} \phi_{\widetilde{U}_{n}, p_{c}, q}^{\mathrm{free}}[0 \leftrightarrow x]=n^{1-\frac{3}{2} \alpha(q, \pi)+o(1)},
$$

so that this quantity can indeed be larger or equal to 1 provided that $q \leq 3$.

The previous discussion remained at the level of predictions and does not seem to provide a good strategy for the proof since it would require to prove much more than we wish to get. A very good news is that the reasoning leading to (3.5) can indeed be applied to $\widetilde{U}_{n}$ instead of $U_{n}$ to give that for $q \leq 3$, there exists $c=c(q)>0$ such that for any $n \geq 1$,

$$
\sum_{x \in \partial \Lambda_{n}} \phi_{\widetilde{U}_{n}, p_{c}, q}^{\text {free }}[0 \leftrightarrow x] \geq c .
$$

Since $\widetilde{U}_{n}$ is a subset of $\mathbb{Z}^{2}$, the comparison between boundary conditions implies that for any $q \leq 3$.

$$
\sum_{x \in \mathbb{Z}^{2}} \phi_{p_{c}, q}^{\text {free }}[0 \leftrightarrow x]=\infty,
$$

thus extending the result to every $q \leq 3$.

This reasoning does not directly extend to $q>3$ since $\frac{3}{2} \alpha(q, \pi)>1$ in this case. Nevertheless, one could consider a graph generalizing $\mathbb{H}$ and $\mathbb{S}$ with a "larger opening than $2 \pi$ " at 0 . In fact, one may even consider a graph with "infinite opening" at 0 by considering subgraphs of the universal cover $\mathbb{U}$ of the plane minus a face of $\mathbb{Z}^{2}$, see Fig. 12. This is what was done in [Dum12]. The drawback of taking this set $\mathbb{U}$ is that it is not a subset of $\mathbb{Z}^{2}$ anymore. Thus, one has to translate the information obtained for the FK percolation on $\mathbb{U}$ into information for the FK percolation on $\mathbb{Z}^{2}$, which is a priori difficult since there is no easy comparison between the two graphs (for instance the comparison between boundary conditions is not sufficient).

To conclude this section, let us mention that the study of lattice models on discrete tori or more generally discrete Riemann surfaces has been the object of a lot of interest in recent years (for instance for dimers and the 


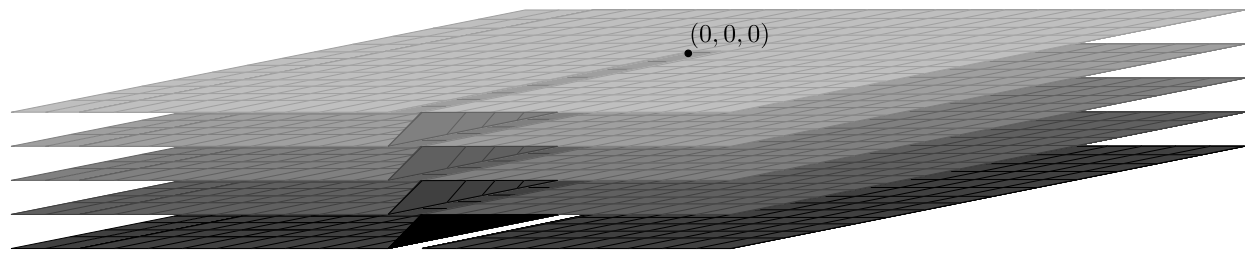

Figure 12. The graph $\mathbb{U}$.

Ising model). Some of the properties of these models are closely related to those on planar graphs, but new interesting features also emerge. The study of lattice models on $\mathbb{U}$ should also be very interesting, and the argument sketched above shows that $\mathbb{U}$ can be useful to understand the models on planar graphs.

3.4. To infinity and beyond: conformal invariance at criticality. We are interested in the rich behavior of FK percolations at the critical value of their continuous phase transition. From now on, we describe the large scale behavior of the macroscopic clusters by rescaling the lattice in such a way that the mesh (i.e. the length of edges) tends to zero. This procedure is called taking the scaling limit. In order to illustrate this procedure, let us consider a very explicit construction.

In this section, $(\Omega, a, b)$ always denotes a simply connected domain $\Omega$ together with two points $a$ and $b$ on its boundary. Also consider Dobrushin domains $\left(\Omega_{\delta}, a_{\delta}, b_{\delta}\right)$ of $\delta \mathbb{Z}^{2}$ converging to $(\Omega, a, b)$ in the Carathéodory sense (see [Dum15] and references therein for details). For smooth boundary for instance, one can for instance consider Dobrushin domains such that $\partial \Omega_{\delta}$ converges in the Hausdorff sense to the boundary of $\Omega$, and that $a_{\delta}$ and $b_{\delta}$ tend to $a$ and $b$ respectively.

In the Dobrushin domains $\left(\Omega_{\delta}, a_{\delta}, b_{\delta}\right)$ with Dobrushin boundary conditions, one can consider the exploration paths $\gamma_{\left(\Omega_{\delta}, a_{\delta}, b_{\delta}\right)}$. These exploration paths form a family of random variables indexed by $\delta>0$ and we may study the convergence of this family as $\delta$ tends to 0 .

Conformal Field Theory predicts that at criticality, $\gamma_{\left(\Omega_{\delta}, a_{\delta}, b_{\delta}\right)}$ converges as $\delta$ tends to 0 to a random, continuous, non-self-crossing curve $\gamma_{(\Omega, a, b)}$ from $a$ to $b$ staying in $\Omega$. Furthermore, the family of curves $\left(\gamma_{(\Omega, a, b)}:(\Omega, a, b)\right)$ is expected to be conformally invariant in the following sense: for any $(\Omega, a, b)$ and any conformal (i.e. holomorphic and one-to-one) map $\psi: \Omega \rightarrow \mathbb{C}$,

$$
\psi\left(\gamma_{(\Omega, a, b)}\right) \text { has the same law as } \gamma_{(\psi(\Omega), \psi(a), \psi(b))} \text {. }
$$

Notice that the conformal invariance of the limit implies the following fact. The random curve obtained by taking the scaling limit of the FK percolation in $(\psi(\Omega), \psi(a), \psi(b))$ has the same law as the image by $\psi$ of the random curve obtained by taking the scaling limit of the FK percolation in $(\Omega, a, b)$. This is clear for $\psi$ which corresponds to a symmetry of the lattice (for instance the rotation by $\frac{\pi}{2} k$ for some $k \in \mathbb{Z}$ ), but this claim implies that 
the property is true for any conformal transformation (therefore in particular for a rotation by any angle).

Fifteen years ago, Schramm [Sch00] proposed a natural candidate for the possible conformally invariant families of continuous non-self-crossing curves. He noticed that interfaces of models further satisfy the domain Markov property which, together with the assumption of conformal invariance, determines a one-parameter family of possible curves: the Stochastic Loewner Evolutions (SLE, which are now known as the Schramm-Loewner Evolutions). Let us briefly describe this object now (see [Law05, Wer04, Wer05] for comprehensive expositions). We start by recalling the definition of a Loewner chain.

Set $\mathbb{H}$ to be the upper half-plane $\mathbb{R} \times(0, \infty)$. Fix a simply connected subdomain $H$ of $\mathbb{H}$ such that $\mathbb{H} \backslash H$ is compact. Riemann's mapping theorem guarantees the existence of a conformal map from $H$ onto $\mathbb{H}$. Moreover, there are a priori three real degrees of freedom in the choice of the conformal map, so that it is possible to fix its asymptotic behavior as $z$ goes to $\infty$. Let $g_{H}$ be the unique conformal map from $H$ onto $\mathbb{H}$ such that

$$
g_{H}(z):=z+\frac{C}{z}+O\left(\frac{1}{z^{2}}\right) .
$$

(The proof of the existence of this map is not completely obvious and requires Schwarz's reflection principle.)

Let $\left(W_{t}\right)_{t>0}$ be a continuous real-valued function (one usually requires a few things about this function, but let us omit these technical conditions here). Fix $z \in \mathbb{H}$ and consider the map $t \mapsto g_{t}(z)$ satisfying the following differential equation up to its explosion time:

$$
\partial_{t} g_{t}(z)=\frac{2}{g_{t}(z)-W_{t}}
$$

For every fix $t$, let $H_{t}$ be the set of $z$ for which the explosion time of the differential equation above is strictly larger than $t$. One may verify that $H_{t}$ is a simply connected open set and that $\mathbb{H} \backslash H_{t}$ is compact. Furthermore, the map $z \mapsto g_{t}(z)$ is a conformal map from $H_{t}$ to $\mathbb{H}$.

If there exists a parametrized curve $\left(\Gamma_{t}\right)_{t>0}$ such that for any $t>0, H_{t}$ is the connected component of $\mathbb{H} \backslash \Gamma[0, t]$ containing $\infty$, the curve $\left(\Gamma_{t}\right)_{t>0}$ is called (the curve generating) the Loewner chain with driving process $\left(W_{t}\right)_{t>0}$.

Then, the Loewner chain in $(\Omega, a, b)$ with driving function $\left(W_{t}\right)_{t>0}$ is simply the image of the Loewner chain in $(\mathbb{H}, 0, \infty)$ by a conformal from $(\mathbb{H}, 0, \infty)$ to $(\Omega, a, b)$.

Definition 3.9. For $\kappa>0$ and $(\Omega, a, b), S L E(\kappa)$ is the random Loewner evolution in $(\Omega, a, b)$ with driving process $\sqrt{\kappa} B_{t}$, where $\left(B_{t}\right)$ is a standard Brownian motion.

By construction, the process is conformally invariant, random and fractal (see Fig. 13). In addition, it is possible to study quite precisely the behavior 


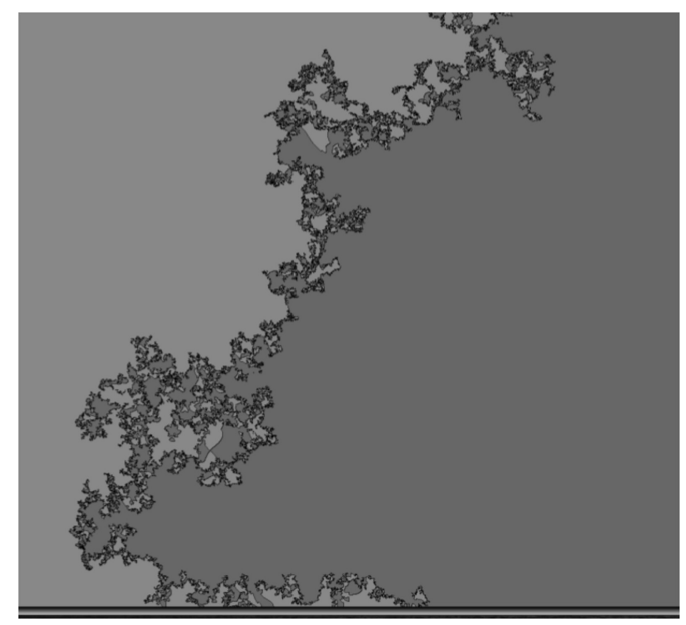

Figure 13. A simulation of $\operatorname{SLE}(6)$ due to V. Beffara.

of SLEs using stochastic calculus and to derive some of their path's properties (e.g. Hausdorff dimension, intersection exponents, etc). Because of the deep understanding of these random curves, the following conjecture is of first importance.

Conjecture $3(\mathrm{Schramm})$. Let $\left(\Omega_{\delta}, a_{\delta}, b_{\delta}\right)$ converging to $(\Omega, a, b)$. The exploration path $\gamma_{\left(\Omega_{\delta}, a_{\delta}, b_{\delta}\right)}$ of the critical $F K$ percolation with parameters $q \in[0,4]$ and $p=p_{c}(q)$ converges weakly to $S L E(\kappa)$ as $\delta$ tends to 0 , where

$$
\kappa=\kappa(q):=\frac{4 \pi}{\pi-\arccos (\sqrt{q} / 2)} .
$$

For completeness, let us mention the topology for the weak convergence mentioned in the conjecture. Let $X$ be the set of continuous parametrized curves and the distance $d\left(\gamma_{1}, \gamma_{2}\right)$ between $\gamma_{1}: I \rightarrow \mathbb{C}$ and $\gamma_{2}: J \rightarrow \mathbb{C}$ defined by

$$
d\left(\gamma_{1}, \gamma_{2}\right)=\min _{\substack{\varphi_{1}:[0,1] \rightarrow I \\ \varphi_{2}:[0,1] \rightarrow J}} \sup _{t \in[0,1]}\left|\gamma_{1}\left(\varphi_{1}(t)\right)-\gamma_{2}\left(\varphi_{2}(t)\right)\right|,
$$

where the minimization is over increasing bijective functions $\varphi_{1}$ and $\varphi_{2}$. Note that $I$ and $J$ can be equal to $\mathbb{R}_{+} \cup\{\infty\}$. The topology on $(X, d)$ gives rise to a notion of weak convergence for random curves on $X$.

Recently, the case $q=2$ of Schramm's conjecture was settled by Chelkak, Duminil-Copin, Hongler, Kemppainen, Smirnov $\left[\mathbf{C D H}^{+} \mathbf{1 4}\right]$. The strategy of the proof is the following. First, prove that the family $\left(\gamma_{\left(\Omega_{\delta}, a_{\delta}, b_{\delta}\right.}\right)_{\delta>0}$ is tight for the weak convergence, see e.g. [KS12, CDH13, DS12a]. Second, use the parafermionic observable with $q=2$ in order to show that any sub-sequential limit of $\left(\gamma_{\left(\Omega_{\delta}, a_{\delta}, b_{\delta}\right.}\right)_{\delta>0}$ is $\operatorname{SLE}(16 / 3)$. This second step is the crucial step of the proof, and it is based on the following result, which represents the main difficulty of the proof of conformal invariance. 
Define the normalized vertex fermionic observable (at criticality) by

$$
f_{\delta}(v)=f_{\delta}\left(\Omega_{\delta}, a_{\delta}, b_{\delta}, p_{c}, 2, v\right):=\frac{1}{\sqrt{2 e_{b}}} \begin{cases}\frac{1}{2} \sum_{u \sim v} F_{\delta}(\{u, v\}) & \text { if } v \in \Omega_{\delta}^{\diamond} \backslash \partial \Omega_{\delta}^{\diamond}, \\ \frac{2}{2+\sqrt{2}} \sum_{u \sim v} F_{\delta}(\{u, v\}) & \text { if } v \in \partial \Omega_{\delta}^{\diamond},\end{cases}
$$

where $F_{\delta}$ is the edge fermionic observable defined in Definition 3.7, and $e_{b}$ is seen as a complex number (note that the modulus of $e_{b}$ is $\frac{\sqrt{2}}{2} \delta$ ).

Theorem 3.10 (Smirnov [Smi10]). Fix $q=2$ and $p=p_{c}(2)$. Let $(\Omega, a, b)$ be a simply connected domain with two marked points on its boundary. Let $\left(\Omega_{\delta}, a_{\delta}, b_{\delta}\right)$ be a family of Dobrushin domains converging to $(\Omega, a, b)$ in the Carathéodory sense. Let $f_{\delta}$ be the normalized vertex fermionic observable in $\left(\Omega_{\delta}, a_{\delta}, b_{\delta}\right)$. We have

$$
f_{\delta}(\cdot) \longrightarrow \sqrt{\phi^{\prime}(\cdot)} \text { when } \delta \rightarrow 0
$$

uniformly on any compact subset of $\Omega$, where $\phi$ is any conformal map from $\Omega$ to the strip $\mathbb{R} \times(0,1)$ mapping a to $-\infty$ and $b$ to $\infty$.

Let us notice that the map $\phi$ is not unique since one could add any real constant to $\phi$, but this modification does not change its derivative.

The function $\sqrt{\phi^{\prime}(\cdot)}$ is the holomorphic solution of a Riemann-Hilbert boundary value problem: its boundary values are orthogonal to the square root of the normal vector to $\partial \Omega$. It does not come as a surprise that the strategy of Smirnov's proof relies on this point. In fact, Smirnov proves that $f_{\delta}$ is a discrete holomorphic function satisfying a proper discretization of the boundary conditions mentioned above. Furthermore, he was able to show that any solution of this "discrete Riemann-Hilbert boundary value problem" must converge to the continuous solution of its continuum counterpart as $\delta$ tends to 0 , thus implying that $f_{\delta}$ tends to $\sqrt{\phi^{\prime}}$.

This result is heavily based on the theory of discrete holomorphic maps. Other than being interesting in themselves, discrete holomorphic functions have also found several applications in geometry, analysis, combinatorics, and probability. We refer the interested reader to the expositions by Lovász [Lov04], Stephenson [Ste05], Mercat [Mer01], Bobenko and Suris [BS08]. This beautiful tool has been applied to several statistical physics models, in particular in the work of Kenyon on dimers [Ken00].

Note that the FK percolation with $q=2$ is coupled to the Ising model. The Ising model itself was proved to be conformally invariant in [CS12], and since then, conformal invariance of many other quantities describing the critical behavior have been derived (crossing probabilities [BDH14], interfaces with other boundary conditions [HK13, Izy15], energy and spin fields [HS13, Hon10, CI13, CHI15]), etc.

Let us mention that the parafermionic observable has even been used off criticality, where it does not satisfy discrete holomorphicity, to study the sub and supercritical phases; see [BD12b, DGP14]. 
We conclude this section by mentioning an important conjecture. Define $f_{\delta}(v)$ as before except that $\sqrt{2 e_{b}}$ is replaced by $\left(2 e_{b}\right)^{\sigma}$ (let us restrict ourselves to non-boundary vertices only).

Conjecture 4 (Smirnov [Smi10]). Fix $q \in(0,4)$ and $p=p_{c}(q)$. Let $(\Omega, a, b)$ be a simply connected domain with two marked points on its boundary. Let $\left(\Omega_{\delta}, a_{\delta}, b_{\delta}\right)$ be a family of Dobrushin domains converging to $(\Omega, a, b)$ in the Carathéodory sense. Let $f_{\delta}$ be the normalized vertex fermionic observable in $\left(\Omega_{\delta}, a_{\delta}, b_{\delta}\right)$. We have

$$
f_{\delta}(\cdot) \longrightarrow \phi^{\prime}(\cdot)^{\sigma} \quad \text { when } \delta \rightarrow 0
$$

uniformly on any compact subset of $\Omega$, where $\phi$ is any conformal map from $\Omega$ to the strip $\mathbb{R} \times(0,1)$ mapping a to $-\infty$ and $b$ to $\infty$.

\section{Critical behavior in dimension 3 and more}

\subsection{Mean field prediction for the order of the phase transition.} Let $q \geq 2$. Consider the Potts model on the complete graph $G_{n}$ given by the vertex set $\{1, \ldots, n\}$ and edge set composed of any pair of vertices in $\{1, \ldots, n\}$. The Hamiltonian on $\mathbb{T}_{q}^{\{1, \ldots, n\}}$ is given by

$$
H_{G_{n}}^{\text {free }}[\sigma]:=-\frac{1}{n} \sum_{x, y=1}^{n}\left\langle\sigma_{x} \mid \sigma_{y}\right\rangle
$$

and the associated measure by $\mu_{G_{n}, \beta}^{\text {free }}$.

Introduce the following functionals: for $h \in \mathbb{R}^{q-1}$, define $G(h)$ to be the Laplace transform of $d \sigma_{0}$ at $h$, i.e.

$$
G(h):=\log \left(\int \exp \left(\left\langle h \mid \sigma_{0}\right\rangle\right) d \sigma_{0}\right)=\log \left(\sum_{v \in \mathbb{T}_{q}} e^{\langle h \mid v\rangle}\right) .
$$

For $m \in \mathbb{R}^{q-1}$, define

$$
\Phi_{\beta, q}(m)=-\frac{\beta}{2}\|m\|^{2}-\inf _{h \in \mathbb{R}^{q-1}}\{G(h)-\langle m, h\rangle\} .
$$

(Recall that $\|\cdot\|$ is the Euclidean norm on $\mathbb{R}^{q-1}$.)

The following fact justifies the introduction of these definitions: the average magnetization $\mathscr{M}\left(G_{n}\right)$ concentrates on values of $m$ minimizing $\Phi_{\beta, q}(m)$ in the following sense: let

$$
M_{\beta}:=\operatorname{argmin}\left\{\Phi_{\beta, q}(m): m \in \mathbb{R}^{q-1}\right\} \subset \mathbb{R}^{q-1},
$$

then for any $\varepsilon>0$,

$$
\lim _{n \rightarrow \infty} \mu_{G_{n}, \beta}^{\text {free }}\left[d\left(\mathscr{M}\left(G_{n}\right), M_{\beta}\right)>\varepsilon\right]=0 .
$$

(Above, $d(x, E)$ is the standard definition of distance from a point to a set.) In particular, if $M_{\beta}$ is a singleton (which is in fact equivalent in our case to $M_{\beta}=\{0\}$ due to the symmetries of $\left.M_{\beta}\right)$, then $\mathscr{M}\left(G_{n}\right)$ must converge to 0 in probability. This happens up to a certain inverse temperature $\beta_{c}(M F) \in$ 
$(0, \infty)$. On the other hand, for $\beta>\beta_{c}(M F)$, the set $M_{\beta}$ contains more than one element. In fact, the function $\Phi_{\beta, q}$ is easy to compute and study so that

- When $q=2$,

$$
M_{\beta}=\left\{-m^{*}(\beta, 2, \infty), m^{*}(\beta, 2, \infty)\right\}=: m^{*}(\beta, 2, \infty) \mathbb{T}_{2}
$$

for $\beta \geq 0$, where $m^{*}(\beta, 2, \infty) \geq 0$ is continuous and equal to 0 if and only if $\beta \leq \beta_{c}(M F)$. In particular, $M_{\beta_{c}(M F)}=\{0\}$.

- When $q \geq 3$,

$$
M_{\beta}=\left\{\begin{array}{ll}
m^{*}(\beta, q, \infty) \mathbb{T}_{q} & \text { if } \beta \neq \beta_{c}(M F) \\
\{0\} \cup m^{*}(\beta, q, \infty) \mathbb{T}_{q} & \text { if } \beta=\beta_{c}(M F)
\end{array},\right.
$$

where $m^{*}(\beta, q, \infty) \geq 0$ equals 0 if and only if $\beta<\beta_{c}(M F)$, is right continuous everywhere and left continuous except at $\beta_{c}(M F)$ where it has a discontinuity.

Because of $(4.1)$, it is natural to interpret $m^{*}(\beta, q, \infty)$ as the spontaneous magnetization of some kind of mean-field infinite-volume Potts measure. The behavior for $q=2$ and $q \geq 3$ is then very different, since the function $\beta \mapsto m^{*}(\beta, q, \infty)$ is continuous in the first case and discontinuous in the second.

4.2. Discontinuity of the phase transition for $q \geq 3$. This section will be devoted to the brief description of two results describing discontinuous phase transitions for the Potts model. They both deal with either $q \gg 1$ or $d \gg 1$.

Case of $q \geq 3$ and $d \gg 1$. Our goal is to compare the behavior of the model to the mean field behavior. We will use the following theorem. Let $\widetilde{\sigma}_{x}:=\sigma_{x}-m^{*}(\beta, q, d) \overrightarrow{1}$ be the spin at $x$ centered around its mean (this definition is useful to speak of so-called truncated correlations).

Theorem 4.1 (Biskup, Chayes [BC03]). Let $d \geq 3$ and $q \geq 2$. Then,

$$
\begin{aligned}
\Phi_{\beta, q}\left(m^{*}(\beta, q, d)\right) & \leq \inf _{m \in \mathbb{R}^{q-1}} \Phi_{\beta, q}(m)+\frac{\beta}{2}\left(\left|\mu_{\beta, q}^{1}\left[\left\langle\sigma_{0} \mid \sigma_{x}\right\rangle\right]\right|^{2}-\left|m^{*}(\beta, q, d)\right|^{2}\right) \\
& \leq \inf _{m \in \mathbb{R}^{q-1}} \Phi_{\beta, q}(m)+\frac{\beta n}{2} I_{d},
\end{aligned}
$$

where $x$ is a neighbor of the origin and $I_{d}$ is the so-called bubble diagram defined by

$$
I_{d}:=\sum_{x \in \mathbb{Z}^{d}} \mu_{\beta, q}^{1}\left[\left\langle\widetilde{\sigma}_{0} \mid \widetilde{\sigma}_{x}\right\rangle\right]^{2} .
$$

We will not use (4.2), but we included here to illustrate how (4.3) is obtained. The proof mainly uses convexity arguments involving the free energy of the model. We refer to [BC03] for details.

Equation (4.3) on the other hand is very useful. Assume for a moment that $I_{d}$ is tiny. Then, (4.3) yields that the spontaneous magnetization $m^{*}(\beta, q, d)$ is almost a minimizer of $\Phi_{\beta, q}$. This implies that $m^{*}(\beta, q, d)$ is 
close to an element of the set $M_{\beta}$. If one fixes $q \geq 3$, one observes that non-zero minimizers of $\Phi_{\beta, q}$ remain bounded away from 0 uniformly in $\beta$. If $I_{d}$ is sufficiently small, this excludes a whole range of possible values for the magnetization, and therefore forces it to be discontinuous provided that we show that $m^{*}(\beta, q, d)$ is not always close to 0 (note that we already know that for $\beta<\beta_{c}, m^{*}(\beta, q, d)$ is equal to 0$)$. But the coupling with FK percolation implies easily that $m^{*}(\beta, q, d)$ converges to 1 as $\beta$ tends to $\infty$, and is therefore close to a non-zero element of $M_{\beta}$. This concludes the proof of the existence of a discontinuity for $m^{*}(\beta, q, d)$, once again provided that $I_{d}$ is tiny.

This last fact is not true in small dimensions. Nevertheless, $I_{d}$ tends to 0 as $d$ tends to infinity (see below), which implies that for any $q \geq 3$, there exists a dimension $d_{c}(q)$ such that for any $d \geq d_{c}(q), m^{*}(\beta, q, d)$ has a discontinuity in $\beta$.

Note that the previous result does not prove that there is a jump in the spontaneous magnetization at $\beta_{c}$, but only at some $\beta$ (which must necessarily be larger or equal to $\beta_{c}$ ). It would be very interesting to prove that this value of $\beta$ is necessarily $\beta_{c}$. This should be the case, since it is predicted that $\beta \mapsto m^{*}(\beta, q)$ is continuous on $\mathbb{R} \backslash\left\{\beta_{c}\right\}$.

As mentioned above, we only need to prove that $I_{d}$ tends to zero as $d$ tends to infinity. This is a fairly direct and simple computation using an upper bound on spin-spin correlations given by the celebrated Infrared Bound, that we discuss now.

Let $G(x, y)$ be the Green function, i.e. the expected time spent at $y$ by a simple random walk starting at $x$. Note that the assumption that $d \geq 3$ guarantees that $G(x, y)$ is finite, since the random-walk is transient in dimension $d \geq 3$.

TheOREM 4.2 (Infrared Bound). Consider the q-state Potts model on $\mathbb{Z}^{d}$ with $d \geq 3$. For any $\beta \in[0, \infty]$ and $v \in\left(\mathbb{R}^{q-1}\right)^{\mathbb{Z}^{d}}$ with finite support,

$$
\sum_{x, y \in \mathbb{Z}^{d}} v_{x} v_{y} \mu_{\beta}^{1}\left[\left\langle\widetilde{\sigma}_{x} \mid \widetilde{\sigma}_{y}\right\rangle\right] \leq \frac{q-1}{2 \beta} \sum_{x, y \in \mathbb{Z}^{d}} v_{x} v_{y} G(x, y)
$$

Note that $G(x, y)$ is the spin-spin correlation for the discrete GFF. Since the $\phi_{d}^{4}$ lattice model interpolates between the Ising and the discrete GFF, it is not so surprising that spin-spin correlations of both models can be compared. The proof of this theorem is based on the so-called Reflection Positivity technique, see Fröhlich, Simon and Spencer [FSS76]. This technique has many applications in different fields of mathematical physics, we refer to [Bis09] and references therein for a more comprehensive study of this subject.

Case of $q \gg 1$ and $d \geq 2$. In this context, the Pirogov-Sinai theory can be harnessed to show that the phase transition is discontinuous. The original result uses Reflection Positivity $[\mathbf{K S 8 2}]$ as well. Since then, the result was 
obtained without the help of Reflection Positivity by [LMR86] and can in fact be extended to FK percolation (also see $[\mathbf{D C}]$ for the $2 \mathrm{D}$ case).

4.3. The case of the Ising model. For many reasons, the Ising model is special among Potts models. One of these reasons is the +/- gauge symmetry: flipping all the spins leaves the measure invariant (for free boundary conditions). In this section, we will explain how this property can be used to derive another geometric representation for the Ising model.

We do not restrict our attention to the nearest neighbor model anymore and treat general ferromagnetic models with interactions $\left(J_{x y}:\{x, y\} \subset \mathbb{Z}^{d}\right)$ satisfying $J_{x y} \geq 0$ for any $x, y$. In this section edges are simply pairs $\{x, y\} \subset$ $\mathbb{Z}^{d}$. The definition of a percolation configuration is modified accordingly.

To adopt standard notation, we will write $\mu_{G, \beta}^{+}$instead of $\mu_{G, \beta}^{1}$. Also note that the Ising model may be defined in infinite-volume thanks to the coupling with the FK percolation ${ }^{1}$ : consider the infinite-volume FK measure with free boundary conditions and assign to each cluster a spin + or - to obtain a measure that we denote by $\mu_{\beta}^{\text {free }}$. Similarly, consider the infinite-volume FK measure with wired boundary conditions and assign to each finite cluster a spin + or - , and to the infinite cluster (if it exists) a spin + , to obtain $\mu_{\beta}^{+}$. We have that $\mu_{G, \beta}^{\text {free }}$ and $\mu_{G, \beta}^{+}$converge to $\mu_{\beta}^{\text {free }}$ and $\mu_{\beta}^{+}$respectively as $G \nearrow \mathbb{Z}^{d}$.

4.3.1. Harvesting the +/- gauge symmetry: the random-current representation. The following perspective on the Ising model's phase transition is driven by the observation that the onset of long range order coincides with a percolation transition in a dual system of currents. This point of view was developed in [Aiz82] and a number of subsequent works. Its advantage comes from the fact that we import the intuition and some tools from the theory of Bernoulli percolation that enable us to draw parallels between proofs in the contexts of Bernoulli percolation and the Ising model.

Definition 4.3. A current $\mathbf{n}$ on $G \subset \mathbb{Z}^{d}$ is a function from $\{\{x, y\} \subset$ $V(G)\}$ to $\mathbb{N}:=\{0,1,2, \ldots\}$. A source of $\mathbf{n}=\left(\mathbf{n}_{x y}:\{x, y\} \subset V(G)\right)$ is a vertex $x$ for which $\sum_{y \in V(G)} \mathbf{n}_{x y}$ is odd. The set of sources of $\mathbf{n}$ is denoted by $\partial \mathbf{n}$. The collection of currents on $G$ is denoted by $\Omega_{G}$. Also set

$$
w_{\beta}(\mathbf{n})=\prod_{\{x, y\} \subset V(G)} \frac{\left(\beta J_{x y}\right)^{\mathbf{n}_{x y}}}{\mathbf{n}_{x y} !}
$$

Let us describe the connection between currents and the Ising model. We start with the random current representation for free boundary conditions.

\footnotetext{
${ }^{1}$ In this case, the FK percolation is not a nearest neighbor model anymore; it can be interpreted as a FK percolation on the complete graph with vertex-set $\mathbb{Z}^{d}$. The edgeweights depend on the pairs $\{x, y\}$ through the same relation as for nearest neighbors, $p_{x y}=1-e^{-\beta J_{x y}}$.
} 
For $\beta>0, G$ a finite graph and $A \subset V(G)$, introduce the quantity

$$
Z^{\text {free }}(G, \beta, A)=\sum_{\sigma \in\{-1,1\} V(G)} \sigma_{A} \prod_{\{x, y\} \subset V(G)} \exp \left[\beta J_{x y} \sigma_{x} \sigma_{y}\right],
$$

where $\sigma_{A}:=\prod_{x \in A} \sigma_{x}$.

Proposition 4.4. Let $\beta>0, G$ be a finite graph and $A \subset V(G)$, then

$$
Z^{\text {free }}(G, \beta, A)=2^{|V(G)|} \sum_{\mathbf{n} \in \Omega_{G}: \partial \mathbf{n}=A} w_{\beta}(\mathbf{n}) .
$$

Proof. Expanding $e^{\beta J_{x y} \sigma_{x} \sigma_{y}}$ for each $\{x, y\}$ to obtain

$$
e^{\beta J_{x y} \sigma_{x} \sigma_{y}}=\sum_{\mathbf{n}_{x y}=0}^{\infty} \frac{\left(\beta J_{x y} \sigma_{x} \sigma_{y}\right)^{\mathbf{n}_{x y}}}{\mathbf{n}_{x y} !}
$$

and substituting this relation in (4.4), one gets

$$
Z^{\text {free }}(G, \beta, A)=\sum_{\mathbf{n} \in \Omega_{G}} w_{\beta}(\mathbf{n}) \sum_{\sigma \in\{-1,1\} V(G)} \prod_{x \in V(G)} \sigma_{x}^{\mathbf{1}_{x \in A}+\sum_{y \in V(G)} \mathbf{n}_{x y}} .
$$

Now, let $x \in V(G)$. Pairing configurations $\sigma$ with the configuration $\sigma^{(x)}$ which coincides with $\sigma$ except at $x$ where the spin is reversed, we see that $\mathbf{1}_{x \in A}+\sum_{y \in V(G)} \mathbf{n}_{x y}$ has to be even for the sum on configurations $\sigma$ not to be equal to 0 . This implies that

$\sum_{\sigma \in\{-1,1\}^{V(G)}} \prod_{x \in V(G)} \sigma_{x}^{\mathbf{1}_{x \in A}+\sum_{y \in V(G)} \mathbf{n}_{x y}}= \begin{cases}0 & \text { if } \sum_{y \in V(G)} \mathbf{n}_{x y} \text { is odd for } \\ & \text { some } x \notin A \text { or even for } \\ & \text { some } x \in A, \\ 2^{|V(G)|} & \text { otherwise. }\end{cases}$

Thus, the definition of a current's source enables one to write

$$
Z^{\text {free }}(G, \beta, A)=2^{|V(G)|} \sum_{\mathbf{n} \in \Omega_{G}: \partial \mathbf{n}=A} w_{\beta}(\mathbf{n})
$$

We deduce that for every $A \subset V(G)$,

$$
\mu_{G, \beta}^{\mathrm{free}}\left[\sigma_{A}\right]=\frac{Z^{\text {free }}(G, \beta, A)}{Z^{\text {free }}(G, \beta, \emptyset)}=\frac{\sum_{\mathbf{n} \in \Omega_{G}: \partial \mathbf{n}=A} w_{\beta}(\mathbf{n})}{\sum_{\mathbf{n} \in \Omega_{G}: \partial \mathbf{n}=\emptyset} w_{\beta}(\mathbf{n})} .
$$

REMARK 4.5. Equation (4.6) implies the first Griffiths' inequality: for any $A \subset V(G)$,

$$
\mu_{G, \beta}^{\text {free }}\left[\sigma_{A}\right] \geq 0
$$

Let us now turn to the random current representation for + boundary conditions. Introduce an additional vertex $\delta \notin \mathbb{Z}^{d}$ called the ghost vertex, and set the coupling $J_{x \delta}=J_{x \delta}(G)$ between it and vertices $x \in V(G)$ to be $\sum_{y \notin V(G)} J_{x y}$. We also set $J_{\delta \delta}=0$. 
Introduce, for $\beta>0, G$ a finite graph and $A \subset V(G)$ the quantity

$$
\begin{aligned}
Z^{+}(G, \beta, A): & =\sum_{\sigma \in\{-1,1\}^{V(G)}} \sigma_{A} \prod_{\{x, y\} \subset V(G)} \exp \left[\beta J_{x y} \sigma_{x} \sigma_{y}\right] \prod_{\substack{x \in V(G) \\
y \notin V(G)}} \exp \left[\beta J_{x y} \sigma_{x}\right] \\
& =\sum_{\sigma \in\{-1,1\}^{V(G)}} \sigma_{A} \prod_{\{x, y\} \subset V(G)} \exp \left[\beta J_{x y} \sigma_{x} \sigma_{y}\right] \prod_{x \in V(G)} \exp \left[\beta J_{x \delta} \sigma_{x}\right] .
\end{aligned}
$$

A development similar to the above yields the following proposition.

Proposition 4.6. Let $\beta>0, G$ be a finite graph and $A \subset V(G)$, then

$$
Z^{+}(G, \beta, A)=2^{|V(G)|} \sum_{\mathbf{n} \in \Omega_{G \cup\{g\}}: \partial \mathbf{n}=A} w_{\beta}(\mathbf{n}) .
$$

Observe that (4.8) differs from (4.5) only through the fact that the summation is over all currents on $G \cup\{g\}$ instead of $G$. Also note that $J_{x \delta}$ depends on $G$.

We obtain a percolation configuration from a current by focusing on the trace $\widehat{\mathbf{n}} \in\{0,1\}^{E(G)}$ of the current $\mathbf{n}$ defined as follows:

$$
\widehat{\mathbf{n}}_{x y}= \begin{cases}1 & \text { if } \mathbf{n}_{x y}>0 \\ 0 & \text { otherwise }\end{cases}
$$

As a consequence, we may speak of $x \stackrel{\widehat{\mathbf{n}}}{\longleftrightarrow} y$ for two vertices $x$ and $y$.

The percolation properties of the random currents are related to the correlations in the Ising model, very much like the percolation properties of FK percolation are related to correlations in the Potts model. In order to illustrate this fact, let us focus on the following proposition.

Proposition 4.7. Let $G$ be a finite graph, $\beta>0$ and $x, y \in G$, then

$$
\mu_{G, \beta}^{\text {free }}\left[\sigma_{x} \sigma_{y}\right]^{2}=\frac{\sum_{\partial \mathbf{n}_{1}=\partial \mathbf{n}_{2}=\emptyset} w_{\beta}\left(\mathbf{n}_{1}\right) w_{\beta}\left(\mathbf{n}_{2}\right) \mathbf{I}\left[x \stackrel{\widehat{\mathbf{n}_{1}+\mathbf{n}_{2}}}{\longleftrightarrow} y\right]}{\sum_{\partial \mathbf{n}_{1}=\partial \mathbf{n}_{2}=\emptyset} w_{\beta}\left(\mathbf{n}_{1}\right) w_{\beta}\left(\mathbf{n}_{2}\right)} .
$$

This proposition follows from the following lemma. Below, $A \Delta B:=(A \backslash$ $B) \cup(B \backslash A)$ denotes the symmetric difference between $A$ and $B$.

Lemma 4.8 (Switching lemma [GHS70, Aiz82]). For any nested pair of finite graph $G$, any pair of vertices $x, y \in V(G)$, any $A \subset V(G)$, and any function $F: \Omega_{G} \rightarrow \mathbb{R}:$ 


$$
\begin{aligned}
& \sum_{\substack{\mathbf{n}_{1} \in \Omega_{G}: \partial \mathbf{n}_{1}=\{x, y\} \\
\mathbf{n}_{2} \in \Omega_{G}: \partial \mathbf{n}_{2}=A}} F\left(\mathbf{n}_{1}+\mathbf{n}_{2}\right) w_{\beta}\left(\mathbf{n}_{1}\right) w_{\beta}\left(\mathbf{n}_{2}\right) \\
& =\sum_{\substack{\mathbf{n}_{1} \in \Omega_{G}: \partial \mathbf{n}_{1}=\emptyset \\
\mathbf{n}_{2} \in \Omega_{G}: \partial \mathbf{n}_{2}=A \Delta\{x, y\}}} F\left(\mathbf{n}_{1}+\mathbf{n}_{2}\right) w_{\beta}\left(\mathbf{n}_{1}\right) w_{\beta}\left(\mathbf{n}_{2}\right) \mathbf{I}\left[x \stackrel{\widehat{\mathbf{n}_{1}+\mathbf{n}_{2}}}{\longleftrightarrow} y\right] .
\end{aligned}
$$

This lemma is very useful when considering sums of two currents. It enables one to switch sources from one current $\mathbf{n}_{1}$ to the other one $\mathbf{n}_{2}$ at the cost of having $x$ connected to $y$ in $\widehat{\mathbf{n}_{1}+\mathbf{n}_{2}}$. This last condition looks necessary, since the sources at $x$ and $y$ in $\mathbf{n}_{1}$ force the existence of a path from $x$ to $y$ along which $\mathbf{n}_{1}$ (and therefore $\mathbf{n}_{1}+\mathbf{n}_{2}$ ) is positive - one may for instance look at the example of $F=1-\mathbf{I}\left[x \stackrel{\widehat{\mathbf{n}_{1}+\mathbf{n}_{2}}}{\longrightarrow} y\right]$. Surprisingly, this is the only constraint one has to take into account. The switching lemma is at the base of almost every application of the random current representation.

Proof. The switching lemma applied to $F=1$ and $A=\{x, y\}$ gives

$$
\begin{aligned}
& \frac{\sum_{\partial \mathbf{n}_{1}=\partial \mathbf{n}_{2}=\emptyset} w_{\beta}\left(\mathbf{n}_{1}\right) w_{\beta}\left(\mathbf{n}_{2}\right) \mathbf{I}\left[x \stackrel{\widehat{\mathbf{n}_{1}+\mathbf{n}_{2}}}{\longrightarrow} y\right]}{\sum_{\partial \mathbf{n}_{1}=\partial \mathbf{n}_{2}=\emptyset} w_{\beta}\left(\mathbf{n}_{1}\right) w_{\beta}\left(\mathbf{n}_{2}\right)} \\
& =\frac{\partial \mathbf{n}_{1}=\partial \mathbf{n}_{2}=\{x, y\}}{\sum_{\partial \mathbf{n}_{1}=\partial \mathbf{n}_{2}=\emptyset} w_{\beta}\left(\mathbf{n}_{1}\right) w_{\beta}\left(\mathbf{n}_{2}\right)} \\
& =\left(\frac{\sum_{\partial \mathbf{n}_{1}=\{x, y\}} w_{\beta}\left(\mathbf{n}_{2}\right)}{\sum_{\partial \mathbf{n}_{1}=\emptyset} w_{\beta}\left(\mathbf{n}_{1}\right)}\right)^{2}=\mu_{G, \beta}^{\text {free }}\left[\sigma_{x} \sigma_{y}\right]^{2} .
\end{aligned}
$$

The random current has been employed extensively in the study of the Ising model. For instance, it was archetypical in the derivation of Aizenman's celebrated result on the triviality of the Ising model for $d \geq 5$ [Aiz82], see below. It was also used in the derivations of several exponents, as well as in the derivation of the sharpness of the phase transition, see [ABF87, DT15]. In this section, we focus on a recent application of the random current representation, which illustrates perfectly the power of a geometric approach to spin-spin correlations.

4.3.2. Continuity of the phase transition for Ising models on $\mathbb{Z}^{d}, d \geq 3$. We are interested in the following result, which provides us with a criterion to show uniqueness of the infinite volume Ising measures.

Theorem 4.9 (Aizenman, DC, Sidoravicius [ADS15]). Let $d \geq 1$ and consider a ferromagnetic Ising model on $\mathbb{Z}^{d}$ with coupling constants $\left(J_{x y}\right)$. If

$$
\lim _{\|x-y\| \rightarrow \infty} \mu_{\beta}^{\text {free }}\left[\sigma_{x} \sigma_{y}\right]=0
$$


then there exists a unique Gibbs state at inverse temperature $\beta$, and in particular $\mu_{\beta}^{\text {free }}=\mu_{\beta}^{+}$.

This fact is utterly wrong for Potts models with $q \geq 3$, as illustrated by the example of the planar Potts model with $q \geq 5$ colors: in such case the phase transition should be discontinuous, hence Theorem 3.1 implies that $\mu_{\beta_{c}}^{1} \neq \mu_{\beta_{c}}^{\text {free }}$, since $\mu_{\beta_{c}}^{1}\left[\sigma_{0}\right] \neq 0$ (since $\left.\phi_{p_{c}, q}^{\text {wired }}[0 \leftrightarrow \infty]>0\right)$ and there is exponential decay of the spin-spin correlations for $\mu_{\beta_{c}}^{\text {free }}$ (since $\phi_{p_{c}, q}^{\text {free }}[x \leftrightarrow y]$ decays exponentially fast in $\|x-y\|)$.

It is tempting to say that, when applied to $\beta=\beta_{c}$, this theorem implies that the phase transition of the Ising model is continuous (observe that if $\mu_{\beta}^{\text {free }}=\mu_{\beta}^{+}$, then $\left.\mu_{\beta}^{+}\left[\sigma_{0}\right]=0\right)$. This is not the case, since (4.9) has a priori no reason to be satisfied at $\beta_{c}$. In fact, the phase transition of the Ising model may be discontinuous, for instance for the model on $\mathbb{Z}$ with $J_{x y}=1 /|x-y|^{2}$, see $[\mathbf{A C C N 8 8}]$. Hence, the previous theorem provides us only with a sufficient condition to decide whether the phase transition is continuous or not.

Now, for the nearest neighbor Ising model on $\mathbb{Z}^{d}(d \geq 3)$, the infrared bound (4.10) can be improved as follows. In the case of the nearest-neighbor Ising model, one can easily deduce from the "averaged" bound given by Theorem 4.2 (which comes from the fact that the proof takes place in the Fourier space) the point-wise one for the free boundary conditions: for any $\beta<\beta_{c}$ and $x, y \in \mathbb{Z}^{d}, \tilde{\sigma}_{x}=\sigma_{x}$ and therefore

$$
\mu_{\beta}^{\text {free }}\left[\sigma_{x} \sigma_{y}\right] \leq \frac{1}{2 \beta} G(x, y)
$$

which, by letting $\beta$ tend to $\beta_{c}$, implies by a classical argument of continuity from below of $\beta \mapsto \mu_{\beta}^{\text {free }}$ that

$$
\mu_{\beta_{c}}^{\text {free }}\left[\sigma_{x} \sigma_{y}\right] \leq \frac{1}{2 \beta_{c}} G(x, y) .
$$

Since the simple random walk is transient on $\mathbb{Z}^{d}$ for $d \geq 3, G(x, y)$ tends to 0 as $\|x-y\|$ tends to infinity. In particular, Theorem 4.9 implies (4.9) and therefore the following result.

Corollary 4.10 ([ADS15]). For $d \geq 3$, the phase transition of the nearest neighbor ferromagnetic Ising model on $\mathbb{Z}^{d}$ is continuous.

Recall that the phase transition is also continuous for $d=2$ (see previous section), so that we conclude that the phase transition of the nearestneighbor ferromagnetic Ising model is always continuous on $\mathbb{Z}^{d}$ for $d \geq 2$.

The proof of Theorem 4.9 is based on the study of the percolation properties of the infinite-volume limit of the random current representation. Let $\mathbf{P}_{G, \beta}^{\text {free }}$ be the law on sourceless currents on $G$ attributing a probability to $\mathbf{n}$ which is proportional to $w_{\beta}(\mathbf{n})$. Similarly, let $\mathbf{P}_{G, \beta}^{+}$be the law on sourceless currents on $G \cup\{g\}$ attributing a probability to $\mathbf{n}$ which is proportional to $w_{\beta}(\mathbf{n})$. Define $\mathbb{P}_{G, \beta}$ to be the law of $\widehat{\mathbf{n}_{1}+\mathbf{n}_{2}}$ restricted to edges of $G$, where 
$\mathbf{n}_{1}$ and $\mathbf{n}_{2}$ are two independent currents with laws $\mathbf{P}_{G, \beta}^{\text {free }}$ and $\mathbf{P}_{G, \beta}^{+}$respectively. This defines a family of percolation measures on finite graphs that gives rise to a percolation measure $\mathbb{P}_{\beta}$ on $\mathbb{Z}^{d}$ by taking the weak limit as $G \nearrow \mathbb{Z}^{d}$.

This measure can be proved to be invariant under translations and ergodic. Also, for any $\beta>0$, the infinite cluster, if it exists, is unique almost surely. As a consequence of these properties,

$$
\lim _{\|x-y\| \rightarrow \infty} \mathbb{P}_{\beta}[x \leftrightarrow y]=0 \quad \Rightarrow \quad(2) \quad \mathbb{P}_{\beta}[0 \leftrightarrow \infty]=0
$$

(uniqueness of the infinite cluster is crucial: the existence of infinitely many infinite clusters would contradict this implication).

Now, the same proof as in Proposition 4.7 implies that for any $x, y \in \mathbb{Z}^{d}$,

$$
\mathbb{P}_{\beta}[x \leftrightarrow y]=\mu_{\beta}^{\text {free }}\left[\sigma_{x} \sigma_{y}\right] \mu_{\beta}^{+}\left[\sigma_{x} \sigma_{y}\right] \leq \mu_{\beta}^{\text {free }}\left[\sigma_{x} \sigma_{y}\right]
$$

The hypothesis of the theorem implies that the right-hand side (1) is satisfied, and therefore that (2) also is. Yet, another application of the switching lemma (with some additional work) implies the existence, for every $x \in \mathbb{Z}^{d}$, of a constant $C_{x}>0$ such that

$$
\mu_{\beta}^{+}\left[\sigma_{0} \sigma_{x}\right]-\mu_{\beta}^{\text {free }}\left[\sigma_{0} \sigma_{x}\right] \leq C_{x} \mathbb{P}_{\beta}[0 \leftrightarrow \infty] .
$$

Thus, (2) implies that $\mu_{\beta}^{+}\left[\sigma_{0} \sigma_{x}\right]=\mu_{\beta}^{\text {free }}\left[\sigma_{0} \sigma_{x}\right]$ for every $x \in \mathbb{Z}^{d}$. This property is classically known to imply that $\mu_{\beta}^{+}=\mu_{\beta}^{-}=\mu_{\beta}^{\text {free }}$. For instance, one may see that it implies the absence of infinite cluster in the FK percolation with wired boundary conditions, and therefore $\mu_{\beta}^{+}=\mu_{\beta}^{-}$(the only difference between the two measures in the coupling would be that the infinite cluster of FK would be + or - respectively, but since there is none, the measures coincide). Thus, $\mu_{\beta}^{\text {free }}$ is sandwiched between $\mu_{\beta}^{+}$and $\mu_{\beta}^{-}$, which are equal, so that $\mu_{\beta}^{\text {free }}=\mu_{\beta}^{+}$.

4.3.3. The four-point function of the Ising model. Let us discuss another application of the random current. The proof of the following proposition is fairly direct using the switching lemma (we leave it as a useful exercise for the reader).

Proposition 4.11 (Aizenman [Aiz82]). Let $\beta>0$ and $G$ be a finite graph. Let $\langle\cdot\rangle$ denote $\mu_{G, \beta}^{\mathrm{free}}$. Then, for any $x_{1}, x_{2}, x_{3}, x_{4} \in V(G)$,

$$
\left\langle\sigma_{x_{1}} \sigma_{x_{2}} \sigma_{x_{3}} \sigma_{x_{4}}\right\rangle-\left\langle\sigma_{x_{1}} \sigma_{x_{2}}\right\rangle\left\langle\sigma_{x_{3}} \sigma_{x_{4}}\right\rangle-\left\langle\sigma_{x_{1}} \sigma_{x_{3}}\right\rangle\left\langle\sigma_{x_{2}} \sigma_{x_{4}}\right\rangle-\left\langle\sigma_{x_{1}} \sigma_{x_{4}}\right\rangle\left\langle\sigma_{x_{2}} \sigma_{x_{3}}\right\rangle
$$

$$
=-2\left\langle\sigma_{x_{1}} \sigma_{x_{3}}\right\rangle\left\langle\sigma_{x_{2}} \sigma_{x_{4}}\right\rangle \cdot \mathbf{P}_{\beta}^{\{1,3\}} \otimes \mathbf{P}_{\beta}^{\{2,4\}}\left[x_{1} \stackrel{\widehat{\mathbf{n}_{1}+\mathbf{n}_{2}}}{\longleftrightarrow} x_{2}, x_{3}, x_{4}\right],
$$

where $\mathbf{P}_{\beta}^{\{a, b\}}$ denotes the law (on currents) attributing probability proportional to $w_{\beta}(\mathbf{n})$ to currents $\mathbf{n}$ with set of sources equal to $\{a, b\}$, and 0 to other currents. 
Above, $\otimes$ means the product of measures. Thus, we see that the connectivity properties of the sum of two independent currents (this time with sources) is once again involved in the estimation of useful truncated $n$-point functions (here the so-called Ursell's four-point function). Let us mention two applications of this result. The first one deals with the planar case.

Corollary 4.12. Let $\beta>0$. Let $G$ be a connected subgraph of $\mathbb{Z}^{2}$ with connected complement. As before, $\langle\cdot\rangle$ denotes $\mu_{G, \beta}^{\mathrm{free}}$. Let $x_{1}, x_{2}, x_{3}$ and $x_{4}$ be four vertices of $\partial G$ found in counter-clockwise order, then

$$
\left\langle\sigma_{x_{1}} \sigma_{x_{2}} \sigma_{x_{3}} \sigma_{x_{4}}\right\rangle=\left\langle\sigma_{x_{1}} \sigma_{x_{2}}\right\rangle\left\langle\sigma_{x_{3}} \sigma_{x_{4}}\right\rangle-\left\langle\sigma_{x_{1}} \sigma_{x_{3}}\right\rangle\left\langle\sigma_{x_{2}} \sigma_{x_{4}}\right\rangle+\left\langle\sigma_{x_{1}} \sigma_{x_{4}}\right\rangle\left\langle\sigma_{x_{2}} \sigma_{x_{3}}\right\rangle .
$$

Note the minus sign in front of the second product on the right-hand side of the equality. The proof follows from the fact that the probability on the right-hand side of (4.11) is equal to 1 . Indeed, a current in $G$ with sources at $x_{1}$ and $x_{3}$ contains a path from $x_{1}$ to $x_{3}$, which must intersect the path from $x_{2}$ to $x_{4}$ present in the current with sources at $x_{2}$ and $x_{4}$. Therefore, $x_{1}, x_{2}, x_{3}$ and $x_{4}$ must necessarily be all connected together in $\widehat{\mathbf{n}_{1}+\mathbf{n}_{2}}$.

The previous result extends to any planar graph, and even to an arbitrary number of vertices $x_{1}, \ldots, x_{2 n}$ (with some mild work). In such case, we obtain a fermionic Wick rule for the $2 n$-point function

$$
\left\langle\sigma_{x_{1}} \cdots \sigma_{x_{2 n}}\right\rangle=\sum_{\pi \in \Pi_{n}} \varepsilon(\pi)\left\langle\sigma_{x_{\pi(1)}} \sigma_{x_{\pi(2)}}\right\rangle \ldots\left\langle\sigma_{x_{\pi(2 n-1)}} \sigma_{x_{\pi(2 n)}}\right\rangle,
$$

where $\Pi_{n}$ is the set of pairings of $\{1, \ldots, 2 n\}$, i.e. the set of permutations $\pi$ such that $\pi(2 j-1)<\pi(2 j)$ for any $j \in\{1, \ldots, n\}$ and $\pi(2 j-1)<\pi(2 j+1)$ for any $j \in\{1, \ldots, n-1\}$. Above, $\varepsilon(\pi)$ is the signature of $\pi$, which can be seen as -1 to the power the parity of the number of intersections of the graph obtained by drawing arcs in $G$ between $\pi(2 j-1)$ and $\pi(2 j)$ for any $j \in\{1, \ldots, n\}$ in such a way that there is a minimal number of intersections.

The second application focuses on the $d \geq 5$ case. There, the quantity on the right-hand side of (4.11) tends to 0 as $x_{1}, x_{2}, x_{3}$ and $x_{4}$ are sent apart. Intuitively, this convergence comes from the fact that the paths from $x_{1}$ to $x_{3}$ in $\mathbf{n}_{1}$ and from $x_{2}$ to $x_{4}$ in $\mathbf{n}_{2}$ behave somehow like random walks. In particular, they do not intersect in dimension $d \geq 5$. Aizenman exploits this intuition and the infrared bound to prove in $[\mathbf{A i z 8 2}]$ that the four-point functions satisfies an asymptotic Wick rule at $\beta_{c}$ when $d \geq 5$. This was also proved by Fröhlich [Frö82] using a random-walk representation [BFS82] of the Ising model à la Symanzik [Sym66].

Theorem 4.13 (Aizenman, Fröhlich [Aiz82, Frö82]). Let $d \geq 5$ and $\beta=\beta_{c}$ and let $\langle\cdot\rangle=\mu_{\beta_{c}}^{\text {free }}$. Then,

$$
\left\langle\sigma_{x_{1}} \sigma_{x_{2}} \sigma_{x_{3}} \sigma_{x_{4}}\right\rangle \sim\left\langle\sigma_{x_{1}} \sigma_{x_{2}}\right\rangle\left\langle\sigma_{x_{3}} \sigma_{x_{4}}\right\rangle+\left\langle\sigma_{x_{1}} \sigma_{x_{3}}\right\rangle\left\langle\sigma_{x_{2}} \sigma_{x_{4}}\right\rangle+\left\langle\sigma_{x_{1}} \sigma_{x_{4}}\right\rangle\left\langle\sigma_{x_{2}} \sigma_{x_{3}}\right\rangle .
$$

Above, $\sim$ means that the ratio of the left and right hand sides tends to 1 as the minimal distance between $x_{1}, x_{2}, x_{3}$ and $x_{4}$ tends to infinity.

This theorem easily implies that the model has a trivial scaling limit. 
Acknowledgements. This work was supported by a grant from the Swiss FNS, by the ERC AG COMPASP and the NCCR SwissMap also funded by the Swiss FNS.

\section{References}

[ABF87] M. Aizenman, D. J. Barsky, and R. Fernández, The phase transition in a general class of Ising-type models is sharp, J. Statist. Phys. 47 (1987), no. 3-4, 343-374. MR 894398 (89f:82013)

[ACCN88] M. Aizenman, J. T. Chayes, L. Chayes, and C. M. Newman, Discontinuity of the magnetization in one-dimensional $1 /|x-y|^{2}$ Ising and Potts models, J. Statist. Phys. 50 (1988), no. 1-2, 1-40. MR 939480 (89f:82072)

[ADS15] M. Aizenman, H. Duminil-Copin, and V. Sidoravicius, Random currents and continuity of Ising model's spontaneous magnetization, Communications in Mathematical Physics 334 (2015), 719-742.

[AF88] M. Aizenman and R. Fernández, Critical exponents for long-range interactions, Lett. Math. Phys. 16 (1988), no. 1, 39-49. MR 958462 (89h:82041)

[Aiz82] M. Aizenman, Geometric analysis of $\varphi^{4}$ fields and Ising models. I, II, Comm. Math. Phys. 86 (1982), no. 1, 1-48. MR 678000 (84f:81078)

[Bax71] R. J. Baxter, Generalized ferroelectric model on a square lattice, Studies in Appl. Math. 50 (1971), 51-69. MR 0307646 (46 \#6766)

[Bax73] R. J. Baxter, Potts model at the critical temperature, Journal of Physics C: Solid State Physics 6 (1973), no. 23, L445.

[Bax78] R. J. Baxter, Solvable eight-vertex model on an arbitrary planar lattice, Philos. Trans. Roy. Soc. London Ser. A 289 (1978), no. 1359, 315-346. MR 0479213 (57 \#18660)

[Bax89] R. J. Baxter, Exactly solved models in statistical mechanics, Academic Press Inc. [Harcourt Brace Jovanovich Publishers], London, 1989, Reprint of the 1982 original. MR 998375 (90b:82001)

$\left[\mathrm{BBMdG}^{+} 14\right]$ N. R. Beaton, M. Bousquet-Mélou, J. de Gier, H. Duminil-Copin, and A. J. Guttmann, The critical fugacity for surface adsorption of self-avoiding walks on the honeycomb lattice is $1+\sqrt{2}$, Comm. Math. Phys. 326 (2014), no. 3, 727-754. MR 3173404

[BC03] M. Biskup and L. Chayes, Rigorous analysis of discontinuous phase transitions via mean-field bounds, Comm. Math. Phys. 238 (2003), no. 1-2, 53-93. MR 1989669 (2004f:82016)

[BD12a] V. Beffara and H. Duminil-Copin, The self-dual point of the twodimensional random-cluster model is critical for $q \geq 1$, Probab. Theory Related Fields 153 (2012), no. 3-4, 511-542. MR 2948685

[BD12b] V. Beffara and H. Duminil-Copin, Smirnov's fermionic observable away from criticality, Ann. Probab. 40 (2012), no. 6, 2667-2689. MR 3050513

[BDH14] S. Benoist, H. Duminil-Copin, and C. Hongler, Conformal invariance of crossing probabilities for the Ising model with free boundary conditions, arXiv:1410.3715, 2014.

[BFS82] D. Brydges, J. Fröhlich, and T. Spencer, The random walk representation of classical spin systems and correlation inequalities, Comm. Math. Phys. 83 (1982), no. 1, 123-150. MR 648362

[BH57] S. R. Broadbent and J. M. Hammersley, Percolation processes. I. Crystals and mazes, Proc. Cambridge Philos. Soc. 53 (1957), 629-641. MR 0091567 $(19,989 \mathrm{e})$

[Bis09] M. Biskup, Reflection positivity and phase transitions in lattice spin models, Methods of contemporary mathematical statistical physics, Lecture 
Notes in Math., vol. 1970, Springer, Berlin, 2009, pp. 1-86. MR 2581604 (2011a:82016)

[BS08] A. I. Bobenko and Y. B. Suris, Discrete differential geometry, Graduate Studies in Mathematics, vol. 98, American Mathematical Society, Providence, RI, 2008, Integrable structure. MR 2467378 (2010f:37125)

[CDH13] D. Chelkak, H. Duminil-Copin, and C. Hongler, Crossing probabilities in topological rectangles for the critical planar FK-Ising model, arXiv:1312.7785, 2013.

$\left[\mathrm{CDH}^{+} 14\right]$ D. Chelkak, H. Duminil-Copin, C. Hongler, A. Kemppainen, and S. Smirnov, Convergence of Ising interfaces to Schramm's SLE curves, C. R. Acad. Sci. Paris Math. 352 (2014), no. 2, 157-161.

[CHI15] D. Chelkak, C. Hongler, and K. Izyurov, Conformal invariance of spin correlations in the planar Ising model, Ann. of Math. (2) 181 (2015), no. 3, 1087-1138. MR 3296821

[CI13] D. Chelkak and K. Izyurov, Holomorphic spinor observables in the critical Ising model, Comm. Math. Phys. 322 (2013), no. 2, 303-332. MR 3077917

[CS12] D. Chelkak and S. Smirnov, Universality in the 2D Ising model and conformal invariance of fermionic observables, Invent. Math. 189 (2012), no. 3, 515-580. MR 2957303

[DC] H. Duminil-Copin, A new proof of first order phase transition a new proof of first order phase transition for the planar random-cluster and Potts models with $q \gg 1$, preprint.

[DGP14] H. Duminil-Copin, C. Garban, and G. Pete, The near-critical planar FKIsing model, Comm. Math. Phys. 326 (2014), no. 1, 1-35. MR 3162481

[DHN11] H. Duminil-Copin, C. Hongler, and P. Nolin, Connection probabilities and $R S W$-type bounds for the two-dimensional FK Ising model, Comm. Pure Appl. Math. 64 (2011), no. 9, 1165-1198. MR 2839298 (2012f:60332)

[DRT15] H. Duminil-Copin, A. Raoufi, and V. Tassion, A new computation of the critical point for the planar random-cluster model with $q \geq 1$, in preparation, 2015.

[DS12a] H. Duminil-Copin and S. Smirnov, Conformal invariance of lattice models, Probability and statistical physics in two and more dimensions, Clay Math. Proc., vol. 15, Amer. Math. Soc., Providence, RI, 2012, pp. 213-276. MR 3025392

[DS12b] H. Duminil-Copin and S. Smirnov, The connective constant of the honeycomb lattice equals $\sqrt{2+\sqrt{2}}$, Ann. of Math. (2) 175 (2012), no. 3, 16531665. MR 2912714

[DST15] H. Duminil-Copin, V. Sidoravicius, and V. Tassion, Continuity of the phase transition for planar random-cluster and Potts models with $1 \leq q \leq 4$, arXiv:1505.04159, 2015.

[DT15] H. Duminil-Copin and V. Tassion, A new proof of the sharpness of the phase transition for Bernoulli percolation and the Ising model, arXiv:1502.03050, 2015 .

[Dum12] H. Duminil-Copin, Divergence of the correlation length for critical planar FK percolation with $1 \leq q \leq 4$ via parafermionic observables, Journal of Physics A: Mathematical and Theoretical 45 (2012), no. 49, 494013.

[Dum13] H. Duminil-Copin, Parafermionic observables and their applications to planar statistical physics models, Ensaios Matematicos, vol. 25, Brazilian Mathematical Society, 2013.

[Dum15] H. Duminil-Copin, Geometric representations of lattice spin models, book, Edition Spartacus, 2015. 
[FK72] C. M. Fortuin and P. W. Kasteleyn, On the random-cluster model. I. Introduction and relation to other models, Physica 57 (1972), 536-564. MR 0359655 (50 \#12107)

[Frö82] J. Fröhlich, On the triviality of $\lambda \varphi_{d}^{4}$ theories and the approach to the critical point in $d \geq 4$ dimensions, Nuclear Phys. B 200 (1982), no. 2, 281-296. MR 643591 (83h:81046)

[FSS76] J. Fröhlich, B. Simon, and T. Spencer, Infrared bounds, phase transitions and continuous symmetry breaking, Comm. Math. Phys. 50 (1976), no. 1, 79-95. MR 0421531 (54 \#9530)

[GHS70] R. B. Griffiths, C. A. Hurst, and S. Sherman, Concavity of magnetization of an Ising ferromagnet in a positive external field, J. Mathematical Phys. 11 (1970), 790-795. MR 0266507 (42 \#1412)

[Gla13] A. Glazman, Connective constant for a weighted self-avoiding walk on $\mathbb{Z}^{2}$, Preprint, 2013.

[Gri99] G. R. Grimmett, Inequalities and entanglements for percolation and random-cluster models, Perplexing problems in probability, Progr. Probab., vol. 44, Birkhäuser Boston, Boston, MA, 1999, pp. 91-105. MR 1703126 (2000i:60115)

[Gri06] G. Grimmett, The random-cluster model, Grundlehren der Mathematischen Wissenschaften [Fundamental Principles of Mathematical Sciences], vol. 333, Springer-Verlag, Berlin, 2006. MR 2243761 (2007m:60295)

[HK13] C. Hongler and K. Kytölä, Ising interfaces and free boundary conditions, J. Amer. Math. Soc. 26 (2013), no. 4, 1107-1189. MR 3073886

[Hon10] C. Hongler, Conformal invariance of Ising model correlations, Ph.D. thesis, université de Genève, 2010, p. 118.

[HS13] C. Hongler and S. Smirnov, The energy density in the planar Ising model, Acta Math. 211 (2013), no. 2, 191-225. MR 3143889

[Isi25] E. Ising, Beitrag zur Theorie des Ferromagnetismus., Z. Phys. 31 (1925), $253-258$.

[ISZ88] C. Itzykson, H. Saleur, and J. B. Zuber, Conformal invariance and applications to statistical mechanics, World Scientific, 1988.

[Izy15] K. Izyurov, Smirnov's observable for free boundary conditions, interfaces and crossing probabilities, Comm. Math. Phys. 337 (2015), no. 1, 225-252. MR 3324162

[Ken00] R. Kenyon, Conformal invariance of domino tiling, Ann. Probab. 28 (2000), no. 2, 759-795. MR 1782431 (2002e:52022)

[KS82] R. Kotecký and S. B. Shlosman, First-order phase transitions in large entropy lattice models, Comm. Math. Phys. 83 (1982), no. 4, 493-515. MR 649814 (83i:82033)

[KS12] A. Kemppainen and S. Smirnov, Random curves, scaling limits and loewner evolutions, arXiv:1212.6215, 2012.

[Law05] G. F. Lawler, Conformally invariant processes in the plane, Mathematical Surveys and Monographs, vol. 114, American Mathematical Society, Providence, RI, 2005. MR 2129588 (2006i:60003)

[Len20] W. Lenz, Beitrag zum Verständnis der magnetischen Eigenschaften in festen Körpern., Phys. Zeitschr. 21 (1920), 613-615.

$\left[\mathrm{LMMS}^{+}\right.$91] L. Laanait, A. Messager, S. Miracle-Solé, J. Ruiz, and S. Shlosman, Interfaces in the Potts model. I. Pirogov-Sinai theory of the Fortuin-Kasteleyn representation, Comm. Math. Phys. 140 (1991), no. 1, 81-91. MR 1124260 (93d:82021a)

[LMR86] L. Laanait, A. Messager, and J. Ruiz, Phases coexistence and surface tensions for the Potts model, Comm. Math. Phys. 105 (1986), no. 4, 527-545. MR 852089 (88i:82030) 
[Lov04] L. Lovász, Discrete analytic functions: an exposition, Surveys in differential geometry. Vol. IX, Surv. Differ. Geom., IX, Int. Press, Somerville, MA, 2004, pp. 241-273. MR 2195410 (2007g:30067)

[Mer01] C. Mercat, Discrete Riemann surfaces and the Ising model, Comm. Math. Phys. 218 (2001), no. 1, 177-216. MR 1824204 (2002c:82019)

[Ons44] L. Onsager, Crystal statistics. I. A two-dimensional model with an orderdisorder transition, Phys. Rev. (2) 65 (1944), 117-149. MR 0010315 $(5,280 \mathrm{~d})$

[Pot52] R. B. Potts, Some generalized order-disorder transformations, Proceedings of the Cambridge Philosophical Society, vol. 48, Cambridge University Press, 1952, pp. 106-109.

[Rus78] L. Russo, A note on percolation, Z. Wahrscheinlichkeitstheorie und Verw. Gebiete 43 (1978), no. 1, 39-48. MR 0488383 (58 \#7931)

[Sch00] O. Schramm, Scaling limits of loop-erased random walks and uniform spanning trees, Israel J. Math. 118 (2000), 221-288. MR 1776084 (2001m:60227)

$\left[\mathrm{SFD}^{+} 12\right] \quad$ S. El-Showk, M. F. Paulos, D. Poland, S. Rychkov, D. Simmons-Duffin, and A. Vichi, Solving the $3 d$ ising model with the conformal bootstrap, Physical Review D 86 (2012), no. 2.

[Sim80] B. Simon, Correlation inequalities and the decay of correlations in ferromagnets, Comm. Math. Phys. 77 (1980), no. 2, 111-126. MR 589426 (82e:82004)

[Smi06] S. Smirnov, Towards conformal invariance of 2D lattice models, International Congress of Mathematicians. Vol. II, Eur. Math. Soc., Zürich, 2006, pp. 1421-1451. MR 2275653 (2008g:82026)

[Smi10] S. Smirnov, Conformal invariance in random cluster models. I. Holomorphic fermions in the Ising model, Ann. of Math. (2) 172 (2010), no. 2, 1435-1467. MR 2680496 (2011m:60302)

[Sta68] H. E. Stanley, Dependence of critical properties on dimensionality of spins, Physical Review Letters 20 (1968), no. 12, 589-592.

[Ste05] K. Stephenson, Introduction to circle packing, Cambridge University Press, Cambridge, 2005, The theory of discrete analytic functions. MR 2131318 (2006a:52022)

[SW78] P. D. Seymour and D. J. A. Welsh, Percolation probabilities on the square lattice, Ann. Discrete Math. 3 (1978), 227-245, Advances in graph theory (Cambridge Combinatorial Conf., Trinity College, Cambridge, 1977). MR 0494572 (58 \#13410)

[Sym66] K. Symanzik, Euclidean quantum field theory. I. Equations for a scalar model, J. Mathematical Phys. 7 (1966), 510-525. MR 0187686

[Wer04] W. Werner, Random planar curves and Schramm-Loewner evolutions, Lectures on probability theory and statistics, Lecture Notes in Math., vol. 1840, Springer, Berlin, 2004, pp. 107-195. MR 2079672 (2005m:60020)

[Wer05] W. Werner, SLE, conformal restriction, loops, European Congress of Mathematics, Eur. Math. Soc., Zürich, 2005, pp. 515-528. MR 2185763 (2006j:82021)

Département de Mathématiques, Université de Genève, Genève SWITZERLAND

E-mail address: hugo.duminil@unige.ch 